\title{
A two-layer skin construct consisting of a collagen hydrogel reinforced by a fibrin-coated polylactide nanofibrous membrane
}

This article was published in the following Dove Press journal: International Journal of Nanomedicine

\author{
Marketa Bacakova ${ }^{\mathrm{I}, *}$ \\ Julia Pajorova ${ }^{1,2, *}$ \\ Antonin Broz' \\ Daniel Hadraba ${ }^{1,3}$ \\ Frantisek Lopot ${ }^{3}$ \\ Anna Zavadakova ${ }^{4}$ \\ Lucie Vistejnova ${ }^{4}$ \\ Milan Beno ${ }^{5}$ \\ Ivan Kostic ${ }^{6}$ \\ Vera Jencova ${ }^{7}$ \\ Lucie Bacakova'
}

'Department of Biomaterials and Tissue Engineering, Institute of Physiology of the Czech Academy of Sciences, Prague, Czech Republic; ${ }^{2}$ nd Faculty of Medicine, Charles University, Prague, Czech Republic; ${ }^{3}$ Department of Anatomy and Biomechanics, Faculty of Physical Education and Sport, Charles University, Prague, Czech Republic; ${ }^{4}$ Biomedical Center, Medical Faculty in Pilsen, Charles University, Pilsen, Czech Republic; ${ }^{5}$ nstitute of Experimental Endocrinology, Biomedical Research Center of the Slovak Academy of Sciences, Bratislava, Slovak Republic; ${ }^{6}$ Institute of Informatics, Slovak Academy of Sciences, Bratislava, Slovak Republic; ${ }^{7}$ Department of Chemistry, Technical University of Liberec, Liberec, Czech Republic

*These authors contributed equally to this work

Correspondence: Marketa Bacakova Institute of Physiology of the Czech Academy of Sciences, Videnska 1083, Prague 4, 14220, Czech Republic Tel +420296443765

Email marketa.bacakova@fgu.cas.cz
Background: Repairs to deep skin wounds continue to be a difficult issue in clinical practice. A promising approach is to fabricate full-thickness skin substitutes with functions closely similar to those of the natural tissue. For many years, a three-dimensional (3D) collagen hydrogel has been considered to provide a physiological 3D environment for cocultivation of skin fibroblasts and keratinocytes. This collagen hydrogel is frequently used for fabricating tissue-engineered skin analogues with fibroblasts embedded inside the hydrogel and keratinocytes cultivated on its surface. Despite its unique biological properties, the collagen hydrogel has insufficient stiffness, with a tendency to collapse under the traction forces generated by the embedded cells.

Methods: The aim of our study was to develop a two-layer skin construct consisting of a collagen hydrogel reinforced by a nanofibrous poly-L-lactide (PLLA) membrane pre-seeded with fibroblasts. The attractiveness of the membrane for dermal fibroblasts was enhanced by coating it with a thin nanofibrous fibrin mesh.

Results: The fibrin mesh promoted the adhesion, proliferation and migration of the fibroblasts upwards into the collagen hydrogel. Moreover, the fibroblasts spontaneously migrating into the collagen hydrogel showed a lower tendency to contract and shrink the hydrogel by their traction forces. The surface of the collagen was seeded with human dermal keratinocytes. The keratinocytes were able to form a basal layer of highly mitotically-active cells, and a suprabasal layer.

Conclusion: The two-layer skin construct based on collagen hydrogel with spontaneously immigrated fibroblasts and reinforced by a fibrin-coated nanofibrous membrane seems to be promising for the construction of full-thickness skin substitute.

Keywords: full-thickness skin substitutes, collagen hydrogel, fibroblast and keratinocyte cocultivation, fibrin, nanostructure

\section{Introduction}

The skin is the organ of the human body that is most exposed to external agents. It provides a natural barrier between the surrounding environment and the human internal organs. From the tissue engineering point of view, the most important feature of the skin is its ability to self-remodel and regenerate, and to replace the tissue that is continuously being lost. Anatomically, the skin tissue can be divided into three layers: epidermis, with its main cellular type of keratinocytes; dermis, consisting mainly of fibroblasts; and hypodermis, which is rich in adipose cells. ${ }^{1}$ For many years, tissue engineers have been trying to construct under in vitro 
conditions an appropriate full-thickness skin equivalent that contains all three layers of the skin. However, there are still many limitations.

Two-dimensional (2D) flat materials are commonly used for cell cultivation, but these materials can induce an apical-basal polarity, which can restrict the adhesion and the migration of the cells in the $x-y$ axis, leading to non-physiological behavior of the skin cells, eg fibroblasts. $^{2,3}$ A more complex three-dimensional (3D) microenvironment is therefore necessary for a non-prescribed polarity of the fibroblasts, and also for their spreading and migration in all three dimensions. ${ }^{2}$ Novel 3D biomaterials aim to mimic the physiological environment of natural extracellular matrix (ECM), and thus to ensure better conditions for cell adhesion, spreading, proliferation, migration, matrix protein synthesis, and differentiation. ${ }^{2,4}$

Synthetic materials made of polylactide (PLA) or polycaprolactone (PCL) are commonly processed into fibrous scaffolds by conventional electrospinning, followed by cell seeding. 5,6 Although the synthetic scaffolds are non-toxic and mimic the mechanical and morphological properties of native tissues, they often do not provide sufficient support for cell adhesion and proliferation. The synthetic materials are therefore often modified with biomolecules in order to improve their attractiveness for cells, and thereby to accelerate the healing process. ${ }^{7}$ For example, in our previous studies, nanofibrous PLA membranes were modified with fibrin, a key protein occurring during wound healing, in order to enhance the adhesion and growth of human dermal fibroblasts. ${ }^{8,9}$ Moreover, we found out that two morphologically different structures of fibrin coatings had a significant influence on the cell behavior. ${ }^{10}$ In addition, other studies have shown that collagen-containing composites or collagen-coated nanofibers are more attractive for cells than pure synthetic scaffolds, due to their greater biocompatibility and biodegradability. ${ }^{9,11,12} \mathrm{Fu}$ et al ${ }^{13}$ (2016) found that collagen-coated PCL nanofibers increased the proliferation of skin fibroblasts. Fibrinogen-coated nanofibers decreased the growth of fibroblasts, but they supported the migration and the expression in these cells of $\alpha$-smooth muscle actin, which is a typical marker for fibroblast differentiation into myofibroblasts. It was also found that the PCL/collagen nanofibrous membrane alone did not stimulate keratinocyte migration, while further coating of this membrane with an ultrafine fibrous collagen network significantly increased the cell motility. ${ }^{14}$ Mahjour et al $^{15}$ used a layer-by-layer method to construct PCL/collagen nanofibers with fibroblasts and keratinocytes, and applied this construct to full-thickness wounds on nude mice. Native collagen in any form therefore increases fibroblast chemotaxis, angiogenesis and keratinocyte migration.

The most recent studies have shown that fibroblast behavior is more physiological if the fibroblasts are entrapped in a 3D structure of hydrogels. ${ }^{16,17}$ The hydrogels are able to selfassemble from a liquid monomeric phase to a polymeric mesh network under a certain temperature, $\mathrm{pH}$, and enzymatic activity. ${ }^{18,19}$ The hydrogels are covalently or noncovalently cross-linked, which enables the encapsulation of living cells. ${ }^{20}$ Various nature-derived hydrogels for wound healing and regeneration applications have been described in earlier studies. These hydrogels were derived mainly from collagen, ${ }^{21}$ fibrin, ${ }^{16}$ alginate-gelatin, ${ }^{22}$ hyaluronan-chitosan or chitosan-gelatin, ${ }^{23,24}$ which are molecules similar to the macromolecular-based components of the body. However, one of the most discussed tasks in tissue engineering is the correlation between the stiffness of a hydrogel and the kinetics of cell adhesion and migration, which is related to the degradation and the remodeling time of the material. ${ }^{25,26}$ The degradation time and the remodeling time should be in balance, and both are highly dependent on the behavior of the encapsulated cells. When the cells are entrapped in hydrogels, the surrounding environment changes their gene expression pattern and stimulates biosynthesis of their own ECM molecules, which results in degradation and remodeling of the hydrogels. ${ }^{4,27}$ Various matrix metalloproteases (MMP-1, MMP-2, MMP-8, MMP-13 and MMP-14) are involved in the degradation processes and in the migration of the cells in the hydrogels. ${ }^{17}$ In addition to the degradation, the cells can also synthesize their own molecules of ECM, and in this way they can remodel the hydrogels. ${ }^{22,28}$ The morphology of fibroblasts in $3 \mathrm{D}$ collagen hydrogels varies from bipolar to dendritic in shape, depending on the stiffness and the tension of the hydrogels. Jiang and Grinnell ${ }^{29}$ and Grinnell ${ }^{30}$ also described the mechanical interactions between the collagen hydrogel and the fibroblasts. Entanglement of the fibroblasts into the surrounding matrix moves their forces to the hydrogel, and this results in contraction or compaction of the hydrogels. ${ }^{2930}$ In order to develop a large-size clinically applicable transplant, Braziulis et $\mathrm{al}^{21}$ used plastic compression for a collagen type I hydrogel to increase the stability and to improve the mechanical properties of the material. ${ }^{21}$ Another way to improve the mechanical properties of the hydrogels is to reinforce them with the use of biodegradable synthetic scaffolds, eg poly(lactic-co-glycolide) (PLGA) nanofibrous membranes or knitted meshes. ${ }^{23,31}$ 
In this study, we prepared a two-layer cell construct consisting of a poly-L-lactide (PLLA) nanofibrous membrane coated with a thin nanofibrous fibrin mesh and seeded with human dermal fibroblasts. After the attachment and the initial growth of the fibroblasts, a collagen hydrogel was formed on the fibroblast-seeded membrane. Finally, human epidermal keratinocytes were seeded on top of the collagen hydrogel. We hypothesized that the 3D-structured collagen hydrogel would enable the fibroblasts to migrate into the hydrogel from the membrane, and the collagen hydrogel with naturally immigrated fibroblasts would therefore simulate the skin dermis. The top of the collagen gel with a layer of adhering and growing keratinocytes would simulate the skin epidermis. The biodegradable PLLA nanofibrous membrane would serve as a substrate for the initial attachment and growth of fibroblasts, and as a mechanical support for a soft collagen hydrogel with skin cells. Coating the membrane with a thin nanostructured fibrin mesh was expected to enhance fibroblast adhesion and proliferation, leading to accelerated fibroblast migration into the collagen hydrogel. We supposed that spontaneous migration of the fibroblasts from the membrane into the collagen hydrogel could lower the tendency of these cells to contract and to shrink the gel by their traction forces, which occurs when fibroblasts are directly embedded into the collagen hydrogel during the gelling process. ${ }^{17,32,33}$ The aims of this study were to evaluate the ability of human dermal fibroblasts to migrate into the collagen hydrogel from the nanofibrous membrane, to compare the behavior and the migration of the fibroblast from a non-coated membrane and from a fibrin mesh-coated membrane, and to evaluate the behavior of human epidermal keratinocytes adhering on the collagen hydrogel surface.

\section{Materials and methods}

\section{Preparation of nanofibrous PLLA membranes}

A nanofibrous PLLA membrane was prepared by the electrospinning process. PLLA with a molecular weight of 45,000-55,000 Da (Polyscitech, Akina Inc., Lafayette, IN, USA) was dissolved in a solvent system composed of a mixture of chloroform and ethanol in a ratio of 9:1 (v/v, Penta s.r.o., Prague, Czech Republic) to a final polymer concentration of $10 \mathrm{wt} \%$. The polymer solution was then used for the electrospinning process.
Nanofibrous membranes were prepared using Nanospider NS 1WS500U (Elmarco s.r.o., Liberec, Czech Republic). The resulting fibers $940 \pm 340 \mathrm{~nm}$ in diameter were collected on a spunbond layer at a distance of $190 \mathrm{~mm}$ from the electrospinning string. The collector rolled at a rate of $10 \mathrm{~mm} /$ minute. The applied voltage was $40 \mathrm{kV}$ for the string and $10 \mathrm{kV}$ for the collector. The process was carried out at an ambient temperature of $25^{\circ} \mathrm{C}$ and at relative humidity of $30 \%$.

\section{Cell harvesting and cultivation}

The two-layer construct consisted of human neonatal dermal fibroblasts and human epidermal keratinocytes. The human neonatal dermal fibroblasts were purchased from Lonza (Basel, Switzerland, Cat. No. CC-2509) and were cultivated in Dulbecco's Modified Eagle's Medium (DMEM; Sigma-Aldrich Co., St Louis, MO, USA) with $10 \%$ of fetal bovine serum (FBS; Sebak $\mathrm{GmbH}$, Aidenbach, Germany) and $40 \mu \mathrm{g} / \mathrm{mL}$ of gentamicin (LEK, Ljubljana, Slovenia). The human epidermal keratinocytes were harvested from the skin of a young adult donor with approval of the local ethics committee (Ethics committee, Medical Faculty of Charles University, approval number EK-VP/15/0/ 2017) and after confirmed written informed consent provided by the donor that is in compliance with the Declaration of Helsinki. The skin graft was digested in $0.25 \%$ trypsin solution (Sigma-Aldrich Co.) for 1 hour at $37^{\circ} \mathrm{C}$. After that, the epidermis was separated from the dermis. The epidermis was inserted in a drop of the cultivation medium. After a while, the cells were spontaneously released from the epidermis graft, and they were collected and seeded on mitomycin-inactivated 3T3 mouse embryonic fibroblasts adhered on the bottom of a cultivation flask. The $3 \mathrm{~T} 3$ mouse embryonic fibroblasts were purchased from Sigma-Aldrich Co. (Cat. No. 93061524-1VL). The keratinocytes were cultivated in a mixture of the cell cultivation DMEM medium and Ham's Nutrient Mixture F12 medium (DMEM/F 12, Sigma-Aldrich Co.) in a ratio of $3: 1$. The mixture was supplemented with $10 \%$ of FBS (Sebak GmbH, Germany), $10 \mathrm{ng} / \mathrm{mL}$ epidermal growth factor (EGF, Sigma-Aldrich Co.), $10 \mu \mathrm{g} / \mathrm{mL}$ insulin (Sigma-Aldrich Co.), $0.4 \mu \mathrm{g} / \mathrm{mL}$ hydrocortisone (Sigma-Aldrich Co.), $10^{-10} \mathrm{M}$ cholera toxin (SigmaAldrich Co.), $1000 \mathrm{U} / \mathrm{mL}$ of streptomycin and 0.1 $\mathrm{mg} / \mathrm{mL}$ of penicillin (Sigma-Aldrich Co.). 
The fibroblasts and keratinocytes were cultivated in an incubator at $37^{\circ} \mathrm{C}$ with a humidified atmosphere saturated with $5 \%$ of $\mathrm{CO}_{2}$ in the air.

\section{Preparation of the two-layer skin construct}

A two-layer cell construct was composed of a PLLA nanofibrous membrane pre-seeded with human dermal fibroblasts, a collagen hydrogel and human dermal keratinocytes (Figure 1). The membrane samples were fixed in Cell Crown inserts (Scaffdex Ltd., Tampere, Finland) fitting into 24-well cell culture plates (TPP, Trasadingen, Switzerland) and seeded with human dermal fibroblasts. The fibroblasts were seeded on the membrane at a density of 30,000 cells, and were cultivated in DMEM supplemented with $10 \%$ of FBS (Sebak $\mathrm{GmbH}$, Germany) and $40 \mu \mathrm{g} / \mathrm{mL}$ of gentamicin (LEK, Slovenia). To enhance the adhesion and proliferation of the fibroblasts, the PLLA membrane was coated with a thin nanofibrous fibrin mesh before cell seeding. After 3 days of fibroblast cultivation, the collagen hydrogel was prepared on the fibroblast-seeded membrane, and the fibroblasts started to migrate into the hydrogel. The ability of the fibroblasts to migrate into a collagen hydrogel from a non-coated membrane and from a fibrin-coated PLLA membrane was compared in four time intervals (on days 1, 3, 7 and 14 after collagen preparation, ie on days 4, 6, 10 and 17 after cell seeding). The preparation of the fibrin mesh and the collagen hydrogel is described in detail below in the following sections.
The construct consisting of the nanofibrous fibrin-coated PLLA membrane was then selected for seeding with keratinocytes on top of the collagen hydrogel (Figure 1). The keratinocytes were seeded at a density of 60,000 cells after 4 days of collagen hydrogel preparation (resp. of fibroblast migration). After the keratinocytes had been seeded, the medium was replaced by DMEM/F12 with supplements, as mentioned above. After 2 days of keratinocyte cultivation, the culture medium was further supplemented with 2-phospho-L-ascorbic acid trisodium salt (AA) at a concentration of $50 \mu \mathrm{g} / \mathrm{mL}$, in order to stimulate the cells to synthesize ECM proteins. The medium was changed every 2 days.

\section{Preparation of the fibrin mesh on the nanofibrous membrane}

The fibrin mesh on the membrane was prepared according to our previously published work. ${ }^{10}$ The fibrin was formed by activation of water-soluble human fibrinogen (341576; EMD Millipore, Billerica, MA, USA) with human thrombin (T6884; Sigma-Aldrich Co.). ${ }^{34}$ Briefly, the fibrinogen in a concentration of $10 \mu \mathrm{g} / \mathrm{mL}$, diluted in TRIS buffer (consisting of $50 \mathrm{mM}$ TRIS- $\mathrm{HCl}, 100 \mathrm{nM} \mathrm{NaCl}$ and $2.5 \mathrm{mM} \mathrm{CaCl} 2$ ), was adsorbed on the membrane for 1 hour. After rinsing with TRIS buffer, the adsorbed fibrinogen was activated with thrombin $(2.5 \mathrm{U} /$ $\mathrm{mL}$ in TRIS buffer) for 15 minutes. The thrombin-activated surface was directly treated without TRIS rinsing with a

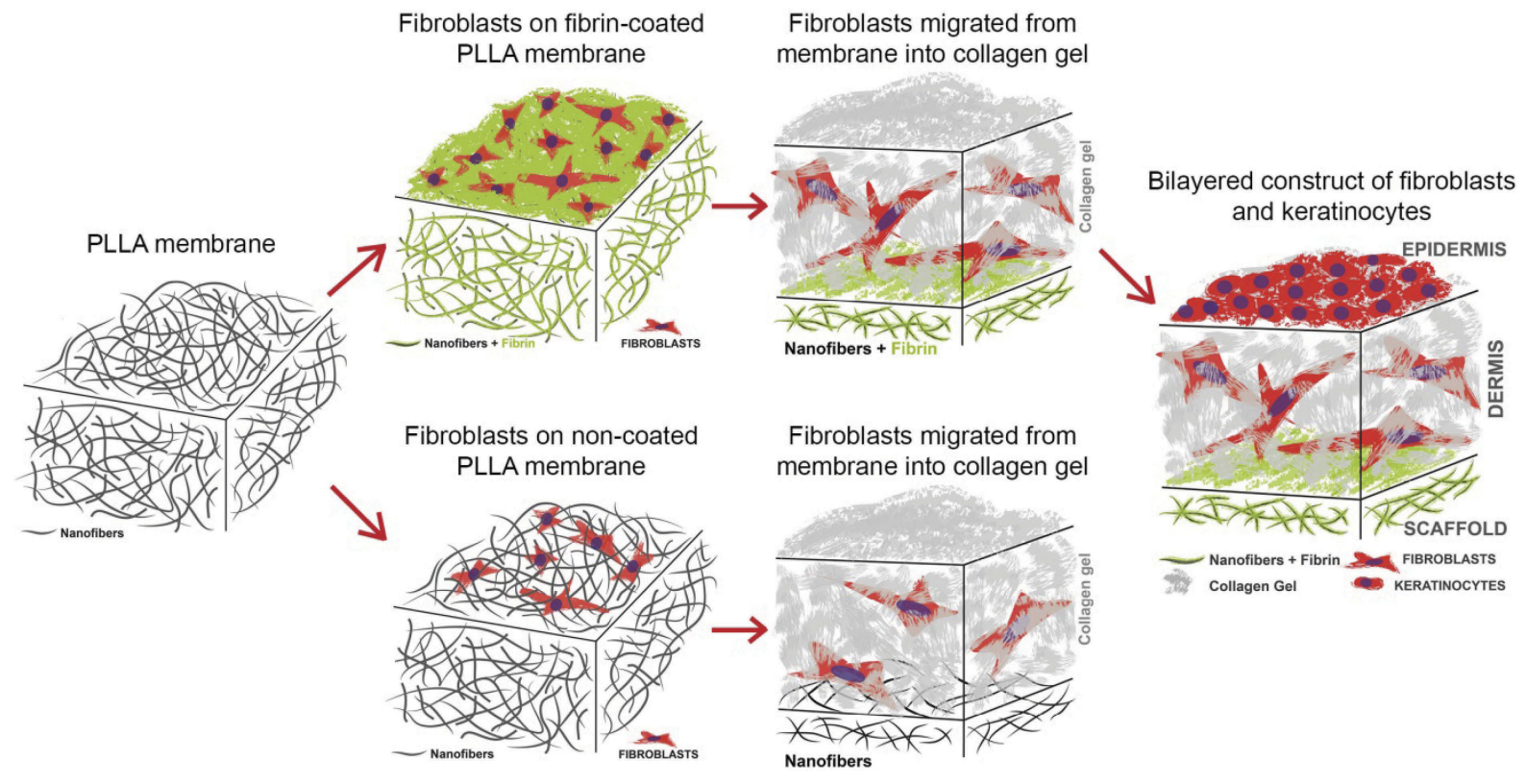

Figure I Scheme of the preparation of the constructs for evaluating the migration of the fibroblasts, consisting of a non-coated membrane or a fibrin-coated PLLA nanofibrous membrane seeded with human dermal fibroblasts and covered with a collagen hydrogel. The construct with the fibrin-coated PLLA nanofibrous membrane was then selected for seeding with keratinocytes and for preparing a bilayered construct simulating the dermis and the epidermis of the natural skin. Abbreviation: PLLA, poly-L-lactide. 
solution of $200 \mu \mathrm{g} / \mathrm{mL}$ of fibrinogen in a TRIS buffer and 0.5 $\mathrm{U} / \mathrm{mL}$ of antithrombin III (Chromogenix, Milano, Italy) in deionized water for 1 hour to form a stable thin fibrin mesh. The antithrombin III blocked the unreacted thrombin remaining on the membrane in order to prevent the formation of a $3 \mathrm{D}$ fibrin gel.

\section{Preparation of the collagen hydrogel on a nanofibrous membrane pre-seeded with fibroblasts}

The type I collagen used for preparing the hydrogel matrix for the migration of the fibroblasts was isolated from rat tails. The tendons were removed from the rat tails, and were digested in $0.1 \mathrm{M}$ acetic acid for 48 hours. The collagen digested in acetic acid was ultracentrifuged at the maximum speed (approx. 46,000 x $g$ ) for 1 hour in order to remove the tissue debris. The supernatant containing the collagen was neutralized with $0.1 \mathrm{M}$ sodium hydroxide and was centrifuged at $1,500 \times \mathrm{g}$ for 10 minutes. The supernatant was removed and the collagen pellet was lyophilized. The lyophilized collagen I was dissolved in $0.02 \mathrm{M}$ acetic acid to a concentration of $5 \mathrm{mg} / \mathrm{mL}$ and was stored as a stock solution.

The collagen hydrogel was prepared on day 3 after seeding of the fibroblasts. For $1 \mathrm{~mL}$ of $3 \mathrm{mg} / \mathrm{mL}$ collagen suspension $600 \mu \mathrm{L}$ of collagen stock solution $(5 \mathrm{mg} / \mathrm{mL})$, $390 \mu \mathrm{L}$ of DMEM (supplemented with $10 \%$ FBS and 40 $\mu \mathrm{g} / \mathrm{mL}$ of gentamicin) and $10 \mu \mathrm{L}$ of sodium bicarbonate (Sigma-Aldrich Co.) were mixed, and a total volume of $400 \mu \mathrm{L}$ was applied on the fibroblast-seeded membrane and was left to polymerize for 20 minutes at $37^{\circ} \mathrm{C}$, in a humidified atmosphere with $5 \%$ of $\mathrm{CO}_{2}$ in the air, to reach $\mathrm{pH}$ around 7.4. The change in $\mathrm{pH}$ from acidic to neutral and $37^{\circ} \mathrm{C}$ caused the collagen to polymerize into a gel. After collagen polymerization, the $1.5 \mathrm{~mL}$ of DMEM (supplemented with $10 \% \mathrm{FBS}$ and $40 \mu \mathrm{g} / \mathrm{mL}$ of gentamicin) was carefully added to the samples.

\section{Collagen hydrogels unconfined compression test}

The collagen hydrogel disks reinforced by a PLLA membrane without cells were prepared under the same conditions as the samples with cells $\left(37^{\circ} \mathrm{C}\right.$, a humidified atmosphere with $5 \%$ of $\mathrm{CO}_{2}, \mathrm{pH} 7.4$ ). Thicker hydrogel disks were required for the unconfined compression test. The hydrogel disks were approximately $3 \mathrm{~mm}$ in thickness for the compression test, and approximately $0.5 \mathrm{~mm}$ in thickness for seeding the cells. The $840 \mu \mathrm{L}$ of collagen stock solution $(5 \mathrm{mg} / \mathrm{mL})$ was diluted in $546 \mu \mathrm{L}$ of DMEM (supplemented with $10 \%$ FBS and $40 \mu \mathrm{g} / \mathrm{mL}$ of gentamicin) and $14 \mu \mathrm{L}$ of sodium bicarbonate (SigmaAldrich Co.). The total volume of $1400 \mu \mathrm{L}$ of collagen solution $(3 \mathrm{mg} / \mathrm{mL})$ was applied on the PLLA membrane without cells. The prepared unconfined samples were 12.6 $\mathrm{mm}$ in diameter, and the compression test was performed on six parallel samples. The fresh samples in PBS were placed between two stainless steel cylinders at $21^{\circ} \mathrm{C}$. The cylinder was attached to the Kistler 9203 force sensor (1000 Hz, sensitivity $0.001 \mathrm{~N}$, range $\pm 10 \mathrm{~N}$, Kistler, Switzerland) and moved in quasi-static mode at $2 \mathrm{~mm} /$ minute (stepper motor PD28-3-1021, Trinamic, Hamburg, Germany). The initial length $1_{0}$ was the length at detecting positive force (height $\mathrm{F} \neq 0=1.82 \pm 0.27 \mathrm{~mm}$ ).

The force and the position data were recalculated into the stress-stain curve reflecting $1_{0}, \varnothing$ and $A_{0}$, respectively. The mechanical properties of the composite material were characterized by the Young's moduli in the linear parts of the stress-strain curves. The data were acquired by the Dewetron data acquisition system (Dewetron, Grambach, Austria) and were analyzed in Matlab 2017a (Mathworks, USA).

\section{Morphology of the fibrin mesh and the collagen hydrogel on the nanofibrous membrane}

The morphology of the fibrin mesh and the collagen hydrogel prepared on a nanofibrous membrane was studied by scanning electron microscopy (SEM). The morphology of the fibrin mesh degraded by cells while they were being cultivated on the fibrin-coated membrane was further observed by fluorescence confocal microscopy.

For SEM, the nanofibrous membrane with fibrin was fixed with a $1 \%$ solution of osmium tetroxide, and the collagen hydrogel was fixed with $4 \%$ paraformaldehyde in PIPES buffer ( $\mathrm{pH} 7$ ) (Sigma-Aldrich Co.). The samples were dehydrated in the standard gradient $(20-40$ minutes in each of $30 \%, 50 \%, 70 \%, 96 \%$, and $100 \%$ ethanol solutions). Samples from absolute alcohol were incubated in the following series of drying solutions: $1: 1$ ethanol/acetone, $100 \%$ acetone, 1:1 acetone/hexamethyldisilazane, $100 \%$ hexamethyldisilazane, with each step taking 20-40 minutes. Air drying of the sample for a period of 24 hours was followed by coating with goldpalladium. The surface morphology and the topography of the fibers were observed using a high-resolution 
scanning electron microscope with a Quanta FEG 250 field emission cathode (FEI, USA). High-vacuum mode, acceleration voltage $10 \mathrm{kV}$ and secondary electrons mode using an Everhart-Thornley detector (ETD) were used for imaging.

The morphology of the fibrin mesh was evaluated by immunofluorescence staining performed on freshly prepared cell-free samples and on samples cultivated with cells in various time intervals. The samples were treated with a solution of $1 \%$ bovine serum albumin and $0.1 \%$ Tween 20 in a phosphate-buffered saline (PBS, all Sigma-Aldrich Co.) for 20 minutes at room temperature, in order to block non-specific binding sites. Then the samples were incubated with a primary antibody against human fibrinogen (A0080; polyclonal rabbit antibody, Dako Denmark A/S, Glostrup, Denmark) diluted in the blocking solution ( $1 \%$ albumin and $0.1 \%$ Tween 20 in PBS) in a ratio of $1: 200$ for 1 hour at $37^{\circ} \mathrm{C}$. After that, the samples were rinsed with PBS and were incubated with a secondary antibody, ie goat anti-rabbit $\mathrm{F}\left(\mathrm{ab}^{\prime}\right) 2$ fragments of $\operatorname{IgG}(\mathrm{H}+\mathrm{L})$, conjugated with Alexa Fluor ${ }^{\circledR} 488$ (A11070; Molecular Probes (Thermo Fisher Scientific), Eugene, OR, USA) diluted in the blocking solution in a ratio of 1:400 for 1 hour at room temperature in the dark. The samples were scanned on a Leica TCS SPE DM2500 upright confocal microscope, objective $40 x / 1.15$ NA oil.

\section{Visualization of the cell morphology and migration in the two-layer construct}

The morphology of the dermal fibroblasts adhered on the nanofibrous membrane and migrated into the collagen hydrogel was studied by staining the filamentous actin (F-actin) cytoskeleton and the cell nucleus. The spreading and the morphology of keratinocytes were evaluated by the same staining, and, in addition, by staining the intermediate filaments containing keratin 14 .

Before staining, the cells were fixed with $4 \%$ paraformaldehyde, and the cell membrane was permeabilized with $0.1 \%$ Triton X-100 (Sigma-Aldrich Co.) diluted in PBS for 30 minutes at room temperature. Then the samples were treated in a solution of $1 \%$ bovine serum albumin and $0.1 \%$ Tween 20 in PBS (all Sigma-Aldrich Co.) to block non-specific binding sites. Subsequently, the F-actin was stained with phalloidin conjugated with tetramethylrhodamine isothiocynate (TRITC) fluorescent dye $(5 \mu \mathrm{g} / \mathrm{mL}$, diluted in PBS, Sigma-Aldrich Co.), and the cell nucleus was stained with Hoechst \#33258 ( $5 \mu \mathrm{g} / \mathrm{ml}$ diluted in PBS, Sigma-Aldrich Co.) for 1 hour at room temperature in the dark. The keratin 14 of the keratinocytes was stained by incubating the samples with primary antibody to human cytokeratin 14 (ab7800, monoclonal mouse antibody, clone LL002, Abcam, Cambridge, United Kingdom) diluted in a blocking solution ( $1 \%$ albumin and $0.1 \%$ Tween 20 in PBS) in a ratio of $1: 200$ for 1 hour at $37^{\circ} \mathrm{C}$. The samples were rinsed with PBS, and were then incubated with a secondary antibody, ie goat anti-mouse $\mathrm{F}(\mathrm{ab}$ ') 2 fragments of $\operatorname{IgG}$ $(\mathrm{H}+\mathrm{L})$, conjugated with Alexa Fluor ${ }^{\circledR} 488$ (A11017; Molecular Probes) diluted in the blocking solution in a ratio of 1:400 for 1 hour at room temperature in the dark. The samples for evaluating fibroblast migration into the collagen hydrogel were scanned on a Leica TCS SPE DM2500 upright confocal microscope, objective 40x/ 1.15 NA oil and 20x/20x/0.75 IMM CORR CS2; FWD 0.66 , zoom $2 x$. The maximal depth of fibroblast migration into the collagen hydrogel from the non-coated or fibrin-coated membranes was measured during scanning on three parallel samples. Statistical significance was evaluated using nonparametric Kruskal-Wallis One Way Analysis of Variance on Ranks, Dunn's Method, statistical significance $(p \leq 0.05)$. Samples of the whole two-layer construct were scanned on a Dragonfly 503 (Andor, Belfast, NI, UK) spinning disk confocal microscope with a Zyla 4.2 PLUS sCMOS camera, objective HC PL APO 20x/0.75 IMM CORR CS2; Free Working Distance $=0.66 \mathrm{~mm}$.

\section{Cell mitochondrial activity}

The proliferation and the viability of the human dermal fibroblasts that adhered on the membrane and that migrated into the collagen hydrogel were determined by the activity of cell mitochondrial enzymes, using the Cell Titer $96^{\circledR}$ AQueous One Solution Cell Proliferation Assay (MTS, Promega Corporation, Madison, WI, USA). The metabolic activity was determined on days 1, 3, 7, and 14 after the collagen hydrogel was prepared.

The collagen hydrogel with immigrated fibroblasts was carefully removed from the membrane, and both parts of the sample were moved into fresh cell culture wells to avoid the influence of cells adhered to the bottom of the well. The metabolic activity of the cells was separately determined in the hydrogel and on the membrane beneath the hydrogel. The non-coated 
membranes and the fibrin-coated membranes without collagen hydrogel seeded with fibroblasts were used as control samples to observe any difference in the metabolic activity of the cells adhered on the two types of membranes. The assay was performed according to the manufacturer's protocol. The amount of formazan dye produced by the cells after 2 hours of incubation was quantified by measuring the absorbance. The absorbance was measured with wavelength $490 \mathrm{~nm}$ and with reference wavelength $700 \mathrm{~nm}$, using a VersaMax ELISA Microplate Reader spectrophotometer (Molecular Devices Corporation, Sunnyvale, CA, USA).

Four parallel samples were used for each experimental group and time interval. The experiment was performed twice. The data was presented as the mean \pm standard deviation (from eight measurements). Statistical significance was evaluated using parametric analysis of variance (ANOVA), with the Tukey post hoc test for pairwise comparison. Values of $p \leq 0.05$ were considered as significant.

\section{An evaluation of the morphology of the collagen hydrogel and its shrinkage by migrating fibroblasts}

The shrinkage of the collagen gel due to migrating fibroblasts was evaluated visually, and the diameter of the collagen hydrogel circle was measured in four time intervals of cell migration, ie on days $1,3,7$, and 14 after the hydrogel was prepared. The diameter of the hydrogel circle was measured on the gel contour marked with several points at the edge of the hydrogel (see below). The freshly prepared collagen samples and the samples incubated without cells for 14 days under cell cultivation conditions were used as controls. Four parallel samples were used for each experimental group and time interval. The diameter of the hydrogel was presented as the mean \pm standard deviation.

\section{Results}

\section{Morphology of the fibrin mesh and the collagen hydrogel on the nanofibrous membrane}

The PLLA membrane consisted of randomly-oriented fibers. The diameter of the fibers varied in a large range, from tens of nanometers to micrometers. The average diameter of the fiber was $940 \mathrm{~nm} \pm 340 \mathrm{~nm}$ (Figure 2A and B).

The fibrin covered the fibers of the membrane, and it also formed a thin homogeneous nanofibrous mesh on the surface of the membrane and among its fibers. The diameter of the fibers of the fibrin mesh ranged from tens of nanometers to approx. $100 \mathrm{~nm}$ (Figure 2C and D).

The nanofibers were formed during the collagen gelling process. They were randomly-oriented in the bulk with a diameter in tens of nanometers. The average diameter of the nanofibers was $47 \pm 7.6 \mathrm{~nm}$ (Figure 2E and F).

\section{Collagen hydrogels unconfined compression test}

The stress strain curves representing the collagen hydrogel disks displayed a linear response up to $21.29 \pm 4.36 \%$
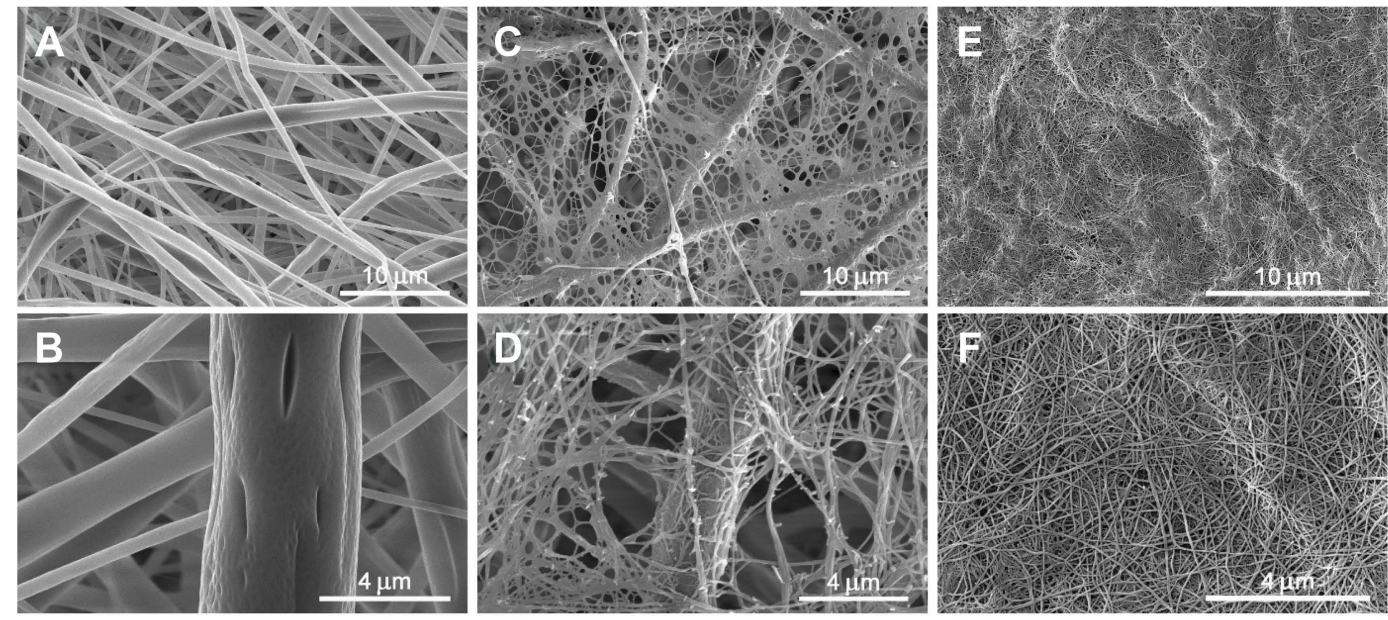

Figure 2 Morphology of a non-modified PLLA membrane (A - magnification 10,000x, B - magnification 30,000x), a fibrin mesh on a PLLA membrane (C - magnification 10,000x, D - magnification 25,000x) and a collagen hydrogel (E - magnification 15 000x, F - magnification 37,587x. Scanning electron microscopy (Quanta FEG 250 highresolution scanning electron microscope).

Abbreviation: PLLA, poly-L-lactide. 
deformation at unconfined compression. The Young's modulus was $3.0 \pm 1.4 \mathrm{kPa}$ in this region. The next linear region was detected from approximately 56.69 $\pm 9.91 \%$ deformation, where the Young's modulus was $89.4 \pm 13.2$ $\mathrm{kPa}$ at unconfined compression; however, structural changes occurred in the sample disks in this region.

\section{Migration of dermal fibroblasts into the collagen hydrogel from fibrin-coated vs non-coated membranes}

We prepared a construct consisting of a pristine, ie noncoated, PLLA nanofibrous membrane or consisting of a membrane coated with a thin nanofibrous fibrin mesh, human dermal fibroblasts and the collagen I hydrogel. The collagen hydrogel was prepared on the fibroblastseeded membrane after 3 days of cell cultivation. We compared the ability of fibroblasts to migrate into the collagen hydrogel from a non-coated membrane and from a fibrin-coated PLLA membrane in four time intervals (on days $1,3,7$, and 14 after collagen preparation, ie on days $4,6,10$, and 17 after cell seeding).

The cell proliferation and viability were determined by measuring the activity of cell mitochondrial enzymes (ie cell metabolic activity). We separately determined the metabolic activity of the fibroblasts migrating in the collagen hydrogel and the fibroblasts adhered on the membrane beneath the hydrogel. Moreover, the metabolic activity of the fibroblasts adhered on the control membranes (non-coated and fibrincoated membranes) without the collagen hydrogel was evaluated in order to see the effect of the fibrin mesh on fibroblast proliferation. There was greater metabolic activity of the cells proliferating on the control fibrin-coated membrane (without collagen) than on the control non-coated membrane (without collagen) (Figure 3, the first pair of columns). We observed these differences in all cultivation time intervals, with statistical significance on day 1. Similarly, the fibrin mesh also increased the fibroblast proliferation on the membrane beneath the collagen (Figure 3, the second pair of columns), with statistical significance on days 1,3 and 7 after the collagen
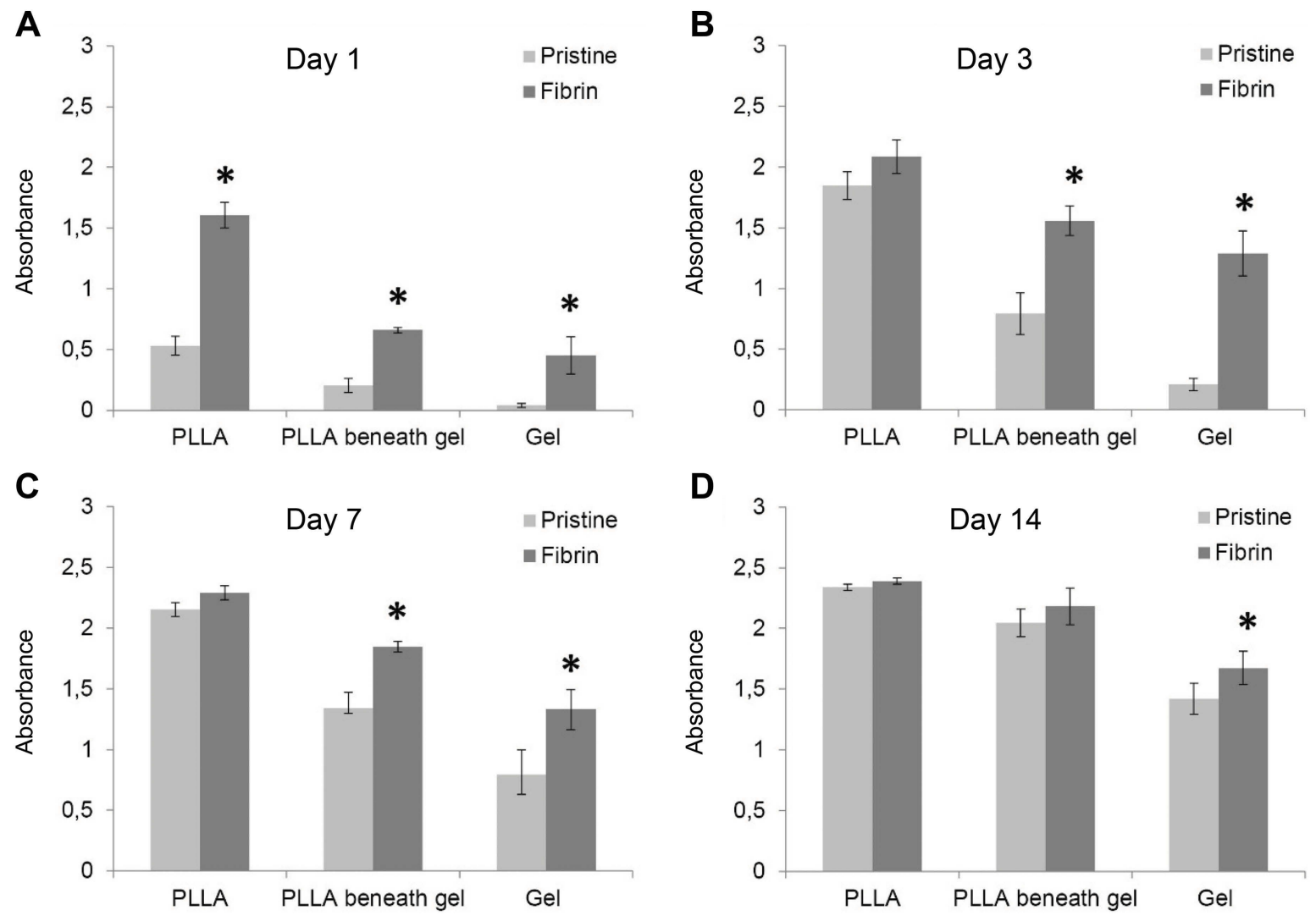

Figure 3 Mitochondrial activity of human dermal fibroblasts migrated into the collagen hydrogel from the non-coated PLLA membrane (Pristine) or from the fibrin-coated (Fibrin) PLLA membrane on day I (A), day $3(\mathbf{B})$, day 7 (C) and day 14 (D) after preparation of the collagen. Cell mitochondrial activity on the control membrane without the hydrogel (PLLA, first pair of columns), separately on the membrane beneath the hydrogel (PLLA beneath gel, second pair of columns) and in the hydrogel (Gel, third pair of columns). Arithmetic mean \pm SD from 8 measurements, ANOVA, Student-Newman-Keuls method, statistical significance ( $p \leq 0.05)$ : $*$ compared with a non-coated membrane (Pristine).

Abbreviation: PLLA, poly-L-lactide. 
hydrogel was prepared (Figure 3A-C). The metabolic activity of the cells migrated into the collagen hydrogel from the fibrincoated membrane was significantly higher than the activity of the cells migrated into the hydrogel from the non-coated membrane in all time intervals (Figure 3, third pair of columns).

The results for cell mitochondrial activity were confirmed by visualization of the cells migrating from the membrane into the collagen hydrogel using immunofluorescent confocal microscopy. The cells migrating into the collagen hydrogel and the cells adhering on the membrane beneath the hydrogel were separately visualized in various time intervals of cell migration (Figure 4). In addition, the fibroblasts adhered on the control membranes (non-coated and fibrin-coated membranes) without the collagen hydrogel were observed (Figure $5)$. Before the preparation of the collagen hydrogel on the membrane after the 3rd day of cell cultivation, the cells were almost in a confluent layer on the fibrin-coated membrane, while on the non-coated membrane, the cells had reached a lower population density (Figure 5, Day 0). On day 1 of cell migration, the cells started to migrate into the collagen hydrogel only from the fibrin-coated membrane. On the non-coated membrane, the cells remained attached to the membrane or to the bottom of the hydrogel (Figure 4, Day 1). In other words, the cells adhering and proliferating on the fibrin-coated membrane started to migrate 1 day after the hydrogel had been prepared, while the cells on the non-coated membrane started migrating later. The supporting influence of the fibrin mesh on the attachment of cells to the membrane and on their proliferation led to a higher number of cells migrating into the collagen hydrogel in all time intervals (Figure 4, the 1st and 2nd rows). In addition, the cells migrated deeper into the hydrogel from the fibrin-coated membrane than from the non-coated membrane, and this difference reached statistical significance on day 3 . The depth of the cell migration increased significantly from the 3rd day to the 7 th day (Figure 6). On day 14, the cells migrated throughout the collagen hydrogel, and they formed a confluent layer on top of the hydrogel, mainly in the case of the fibrin-coated membrane (Figure 4, 1st and 2nd row).

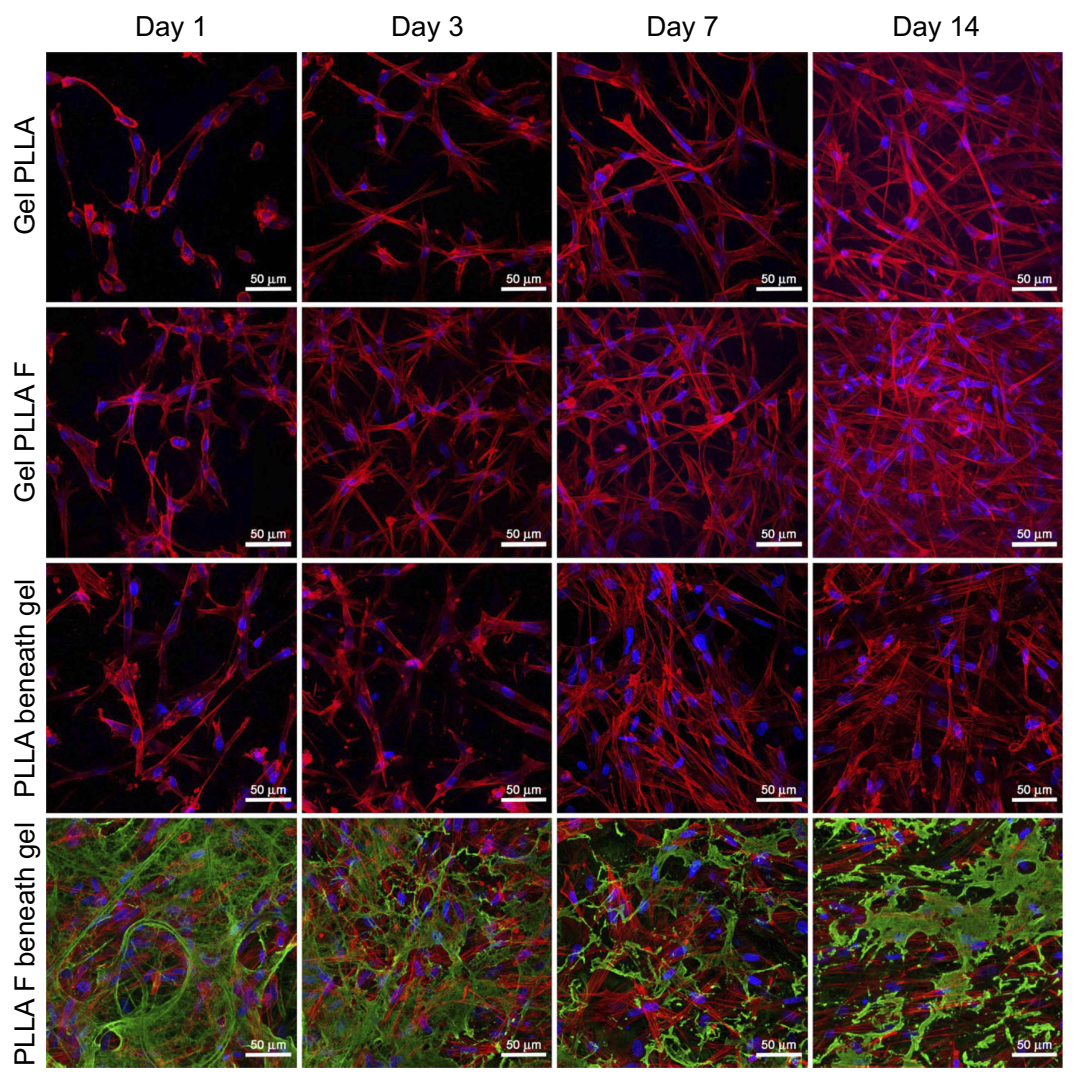

Figure 4 Human dermal fibroblasts in the collagen hydrogel migrated from a non-coated membrane (Gel PLLA) and from a fibrin-coated membrane (Gel PLLA F) for I, 3, 7, and 14 days, or human dermal fibroblasts proliferated on the non-coated membrane (PLLA beneath gel) or on the fibrin-coated membrane (PLLA F beneath gel) beneath the collagen hydrogel for 4, 7, 10 , and 17 days. The fibrin was stained by immunofluorescence (Alexa 488, green). The cells were stained with phalloidin-TRITC (red; F-actin cytoskeleton) and with Hoechst \#33,258 (blue; cell nuclei). Leica TCS SPE DM2500 confocal microscope, 20x/0.75 IMM CORR CS2 zoom 2x or obj. 40x/I.I5 NA oil, maximal intensity projection images.

Abbreviation: PLLA, poly-L-lactide. 

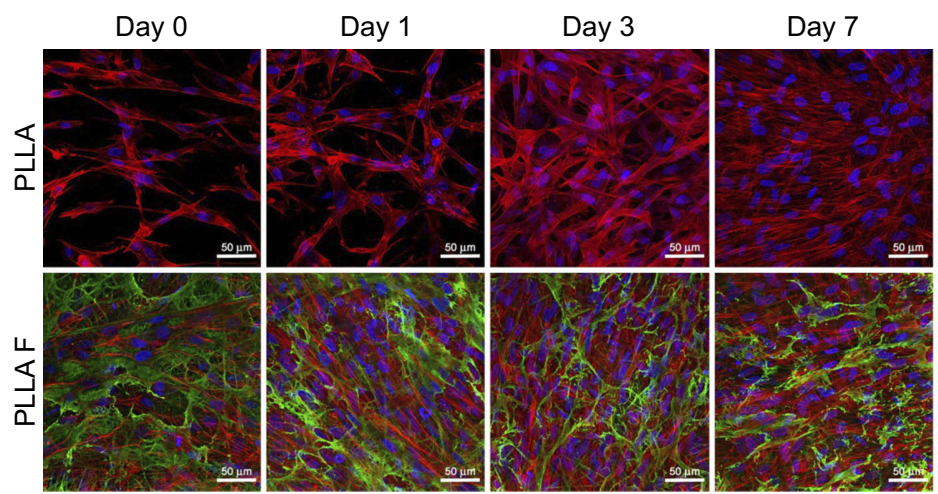

Day 14

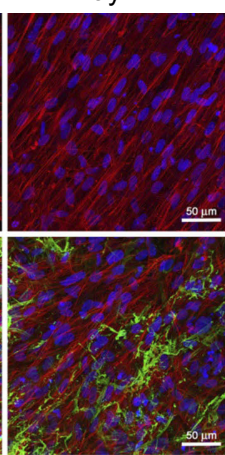

Figure 5 Human dermal fibroblasts on the control non-coated membrane (PLLA) and on the fibrin-coated membranes (PLLA F) without the collagen gel. The cells on the membranes on the 3rd day after they were seeded and before collagen hydrogel preparation (Day 0), and cultivated for I, 3, 7, and I4 additional days (ie 4, 7, I0,and I7 days in total). The fibrin was stained by immunofluorescence (Alexa 488, green). The cells were stained with phalloidin-TRITC (red; F-actin cytoskeleton) and with Hoechst \#33258 (blue; cell nuclei). Leica TCS SPE DM2500 confocal microscope, objective 40x/I.15 NA oil, maximal intensity projection images.

Abbreviation: PLLA, poly-L-lactide.

The cells that remained adhering on the membrane beneath the collagen hydrogel were able to divide and proliferate, and their population increased in time (Figure 4, the 3 rd and 4 th rows). If we compare the cell behavior on the membranes beneath the collagen hydrogel (Figure 4, the 3rd and 4th rows) and on the control membranes (without collagen, Figure 5), the number of adherent cells was significantly higher on the control membranes in all time intervals. On the control fibrin-coated membrane, the cells gradually degraded and reorganized the fibrin mesh. On day 14 , the fibrin mesh was almost degraded, and only residues of it remained on the membrane (Figure 5, 2nd row), while the fibrin coating on the membrane beneath the hydrogel was only slightly altered by the cells (Figure 4, 4th row).

The collagen hydrogel was not considerably contracted and shrunk by the fibroblasts migrating in the collagen over a period of 14 days (Figure 7). The fresh collagen hydrogel prepared on the fibroblast-seeded

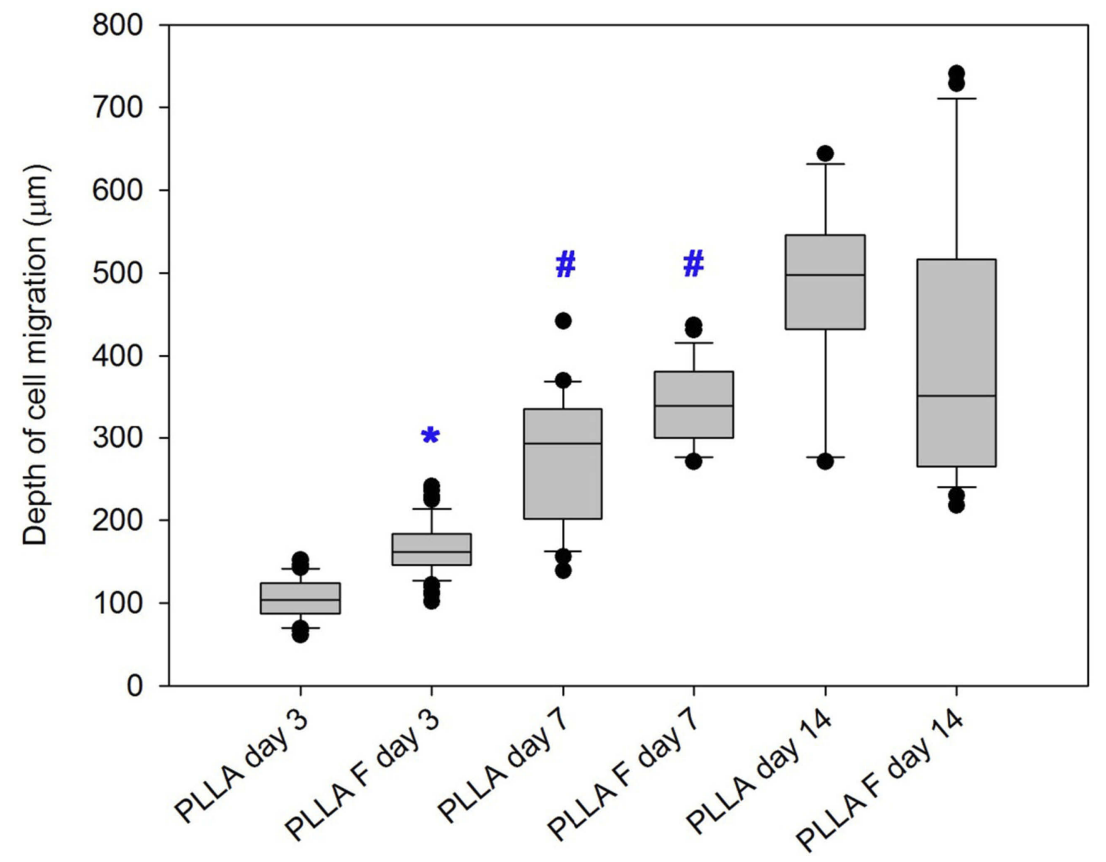

Figure 6 Depth of migration of human dermal fibroblasts into the collagen hydrogel from the non-coated PLLA membrane (PLLA) or from the fibrin-coated PLLA membrane (PLLA + F) on days 3, 7, and I4 of cell migration. Nonparametric Kruskal-Wallis One Way Analysis of Variance on Ranks, Dunn's Method, statistical significance $(p \leq 0.05)$ : *in comparison with the non-coated membrane (PLLA), ${ }^{\#}$ in comparison with day 3. 


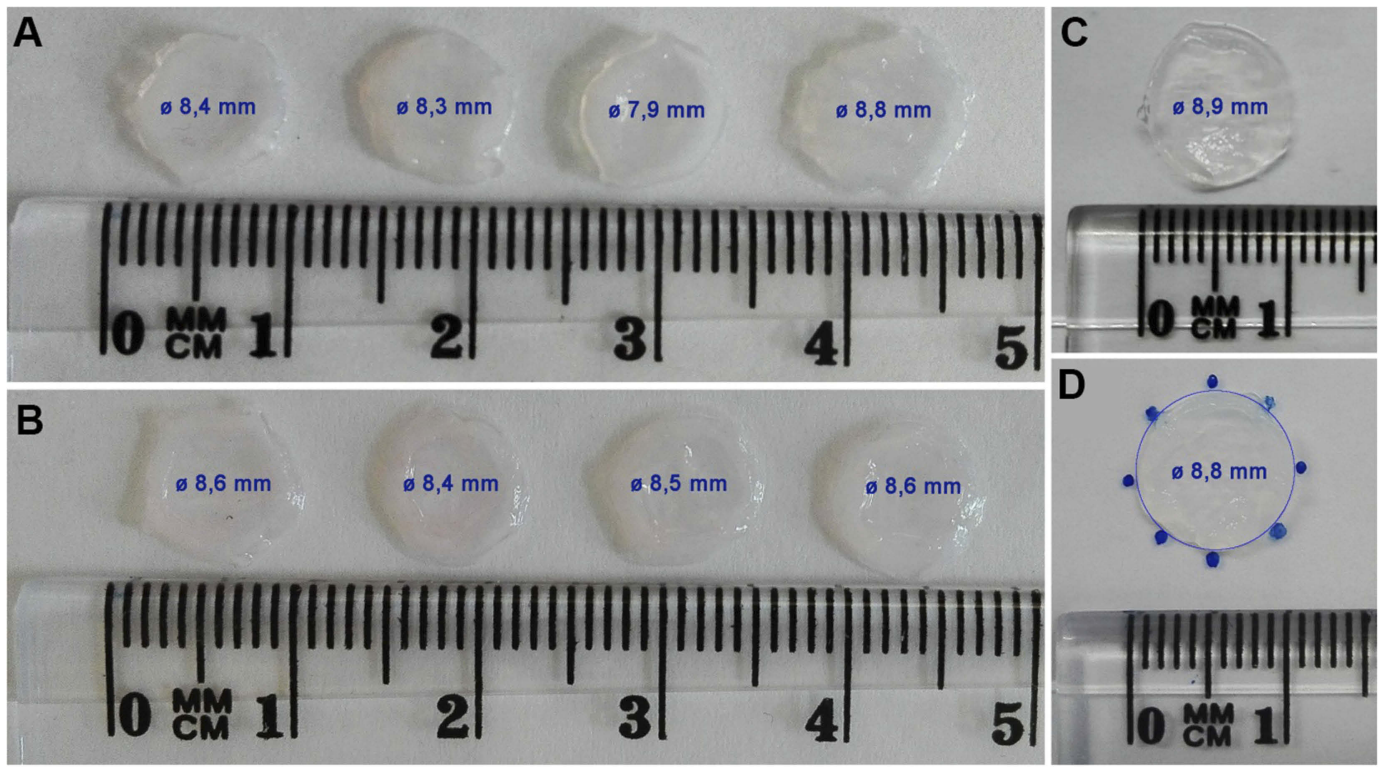

Figure 7 The morphology of the collagen hydrogel with embedded human dermal fibroblasts migrated from the non-coated membrane (A) and from the fibrin-coated membrane (B) for 14 days. The control collagen hydrogel without cells incubated at $37^{\circ} \mathrm{C}, 5 \% \mathrm{CO}_{2}$ for 14 days (C). The measurement principle for hydrogel shrinkage (D): the diameter of the hydrogel circle was measured on the gel contour marked with several points. The value of the diameter $(\varnothing)$ of each hydrogel is displayed inside the hydrogel image, and is presented as mean \pm standard deviation.

membrane was approximately $8.9 \mathrm{~mm}$ in diameter. After 14 days of cell migration, the diameter of the hydrogel reached $8.4 \pm 0.3 \mathrm{~mm}$ (presented as a mean \pm standard deviation). In addition, there was no obvious difference between the remodeling of the collagen hydrogel by the cells migrating from the non-coated membrane (hydrogel diameter was $8.3 \pm 0.4 \mathrm{~mm}$ ) and from the fibrin-coated membrane (hydrogel diameter was $8.5 \pm 0.1 \mathrm{~mm})$.

\section{Creating the two-layer construct consisting of dermal fibroblasts and keratinocytes}

A two-layer construct was prepared using a PLLA nanofibrous membrane coated with a thin nanofibrous fibrin mesh and seeded with human dermal fibroblasts. The collagen hydrogel prepared after 3 days of fibroblast seeding allowed the fibroblasts to migrate inside the gel. The human dermal keratinocytes were seeded on top of the collagen hydrogel after 4 days of fibroblast migration.

Before the keratinocytes were seeded, the fibroblasts were allowed to immigrate into the collagen hydrogel (Figure 8A and $\mathrm{B}$ ). Inside the hydrogel, the cells were spread, and showed a spindle-like morphology with well-developed F-actin microfilaments (Figures 8 and 9), as we also showed in the previous section in Figure 4. After the collagen hydrogel had been seeded with keratinocytes, the fibroblasts continued proliferating inside the hydrogel and migrating through the hydrogel from the bottom to the top (Figure 9). The keratinocytes were able to attach to the collagen hydrogel, and to divide and proliferate throughout the experiment, ie for 14 days. The number of proliferating keratinocytes increased with cultivation time. The cells had already reached a confluent layer on the whole surface of the collagen hydrogel 7 days after keratinocyte seeding (Figure 9C and D). The keratinocytes had well-developed filaments containing cytokeratin 14 (Figure 9). Video S1 shows the keratinocytes and fibroblasts in the collagen hydrogel in individual horizontal sections from the top to the bottom of the collagen hydrogel. On the hydrogel surface, the keratinocytes formed a suprabasal layer of large cells frequently without cell nuclei and a confluent basal layer of small cells that were still dividing and proliferating. The layer of keratinocytes does not allow the fibroblasts to migrate throughout the whole volume of the collagen hydrogel, thus the fibroblasts remained inside the hydrogel without migrating to the surface (Figure 9). If the keratinocytes were not seeded on the collagen hydrogel, the fibroblasts migrated through the whole thickness of the gel and formed a confluent layer on top of the hydrogel (Figure 8E-H).

Figure 10 and Video S2 show the entire two-layer construct after 14 days of keratinocyte cultivation. The bottom of the skin construct is composed of a nanofibrous membrane with a confluent layer of proliferating fibroblasts (red) and with 

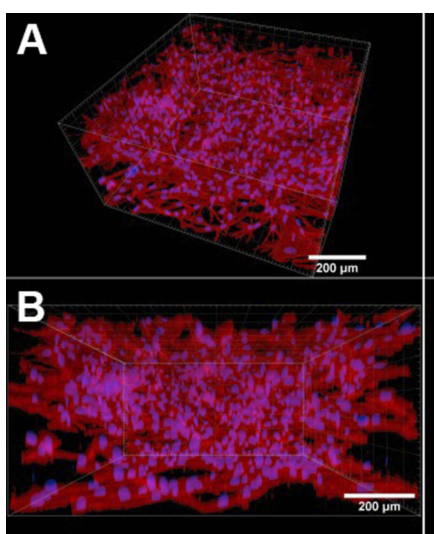

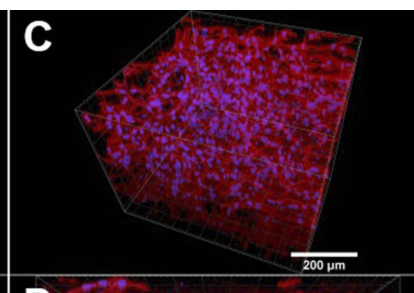

D

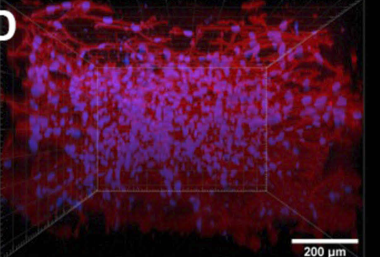

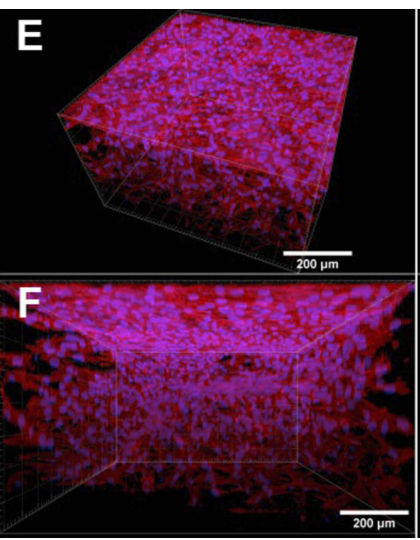

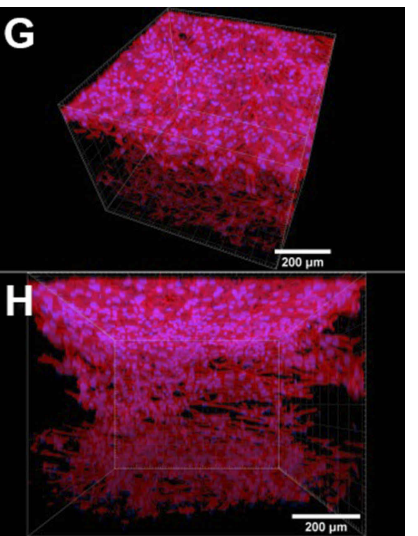

Figure 8 Human dermal fibroblasts in the collagen hydrogel migrated from the fibrin-coated PLLA membrane, without the hydrogel being seeded with keratinocytes (control samples). The fibroblasts migrated from the membrane into the collagen hydrogel for 4 days $(\mathbf{A}$ and $\mathbf{B}), 6$ days $(\mathbf{C}$ and $\mathbf{D})$, II days (E and $\mathbf{F})$, and I8 days (G and $\mathbf{H})$. The fibroblasts were stained with phalloidin-TRITC for the cell F-actin cytoskeleton (red), and with Hoechst \#33258 for the cell nuclei (blue). Dragonfly 503 spinning disk confocal microscope with a Zyla 4.2 PLUS sCMOS camera, objective HC PL APO 20x/0.75 IMM CORR CS2.

Abbreviation: PLLA, poly-L-lactide.

residues of the fibrin mesh (green) degraded by the fibroblasts. The fibroblasts are homogeneously immigrated inside the collagen hydrogel, and the keratinocytes are adhered in the confluent layer on top of the collagen hydrogel (green).

Adding keratinocytes into the construct did not significantly promote shrinkage of the collagen hydrogel. There was a similar slight alteration in the morphology and in the contraction of the collagen hydrogel during cell cultivation when only fibroblasts were seeded into the construct, see our comments in the previous section, and Figure 7.

\section{Discussion}

Many previous studies have shown that the 3D microenvironment of hydrogels provides better physiological conditions than 2D flat substrates for cell spreading, proliferation, migration and differentiation. ${ }^{35}$ The cells embedded in the hydrogels, especially fibroblasts and other mesenchymal cells, tend to be more spread, with a typical spindle-like morphology, and they form a network with cell contacts in all three dimensions. ${ }^{16,17}$ On

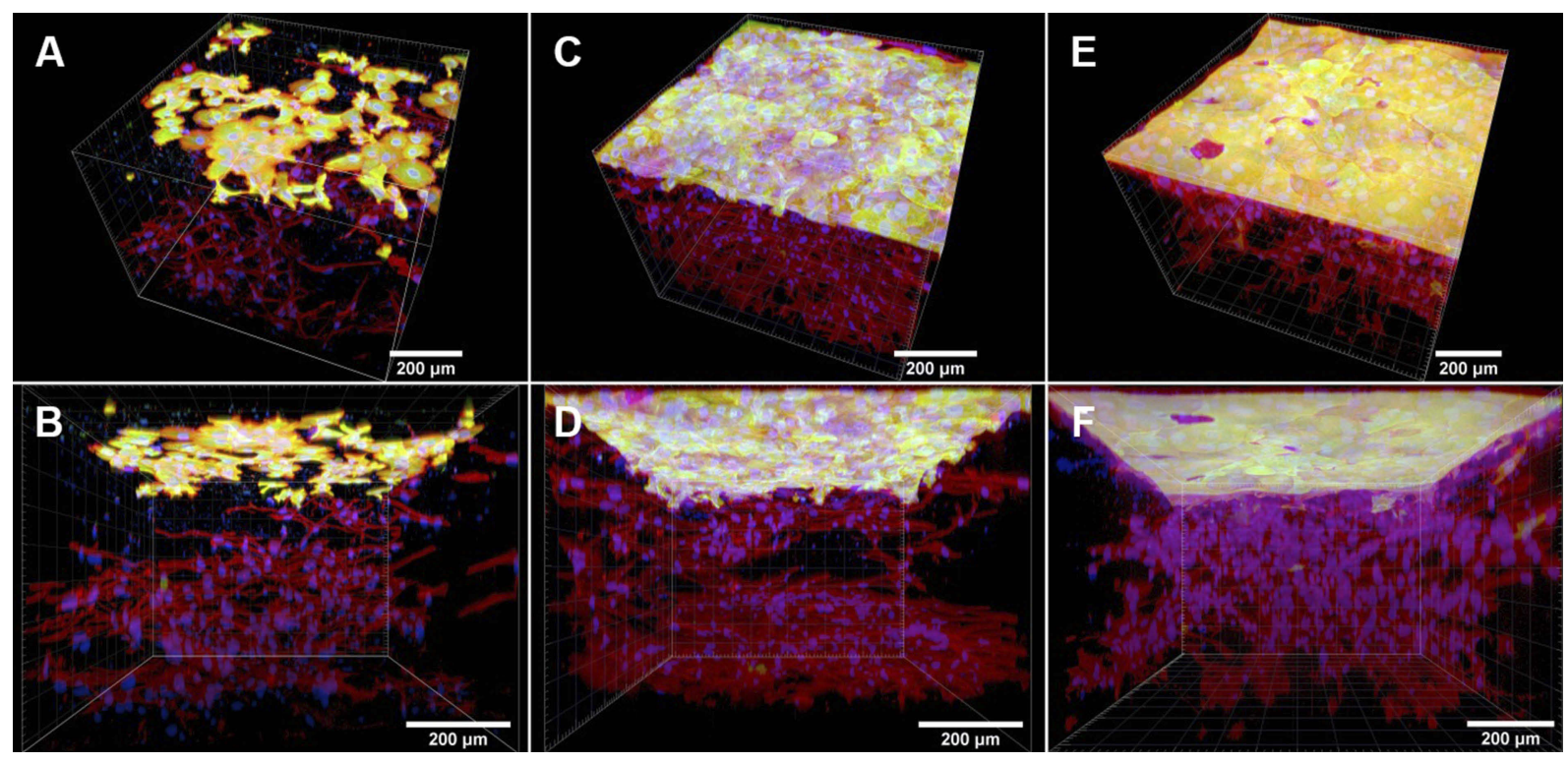

Figure 9 Human dermal fibroblasts in the collagen hydrogel migrated from the fibrin-coated PLLA membrane, and human dermal keratinocytes seeded on top of the hydrogel on day 4 of fibroblast migration. The fibroblasts migrated from the membrane into the collagen hydrogel for a period of 6 days (A and $\mathbf{B})$, II days (C and $\mathbf{D})$ and 18 days ( $\mathbf{E}$ and $\mathbf{F})$. The keratinocytes were cultivated for 2 days ( $\mathbf{A}$ and $\mathbf{B}), 7$ days $(\mathbf{C}$ and $\mathbf{D})$ and 14 days $(\mathbf{E}$ and $\mathbf{F})$. Both cell types were stained with phalloidin-TRITC for the cell F-actin cytoskeleton (red), and with Hoechst \#33258 for the cell nuclei (blue). The cytokeratin 14 in the keratinocytes was stained by immunofluorescence (Alexa 488 , green). Dragonfly 503 spinning disk confocal microscope with a Zyla 4.2 PLUS sCMOS camera, objective HC PL APO 20x/0.75 IMM CORR CS2.

Abbreviation: PLLA, poly-L-lactide. 

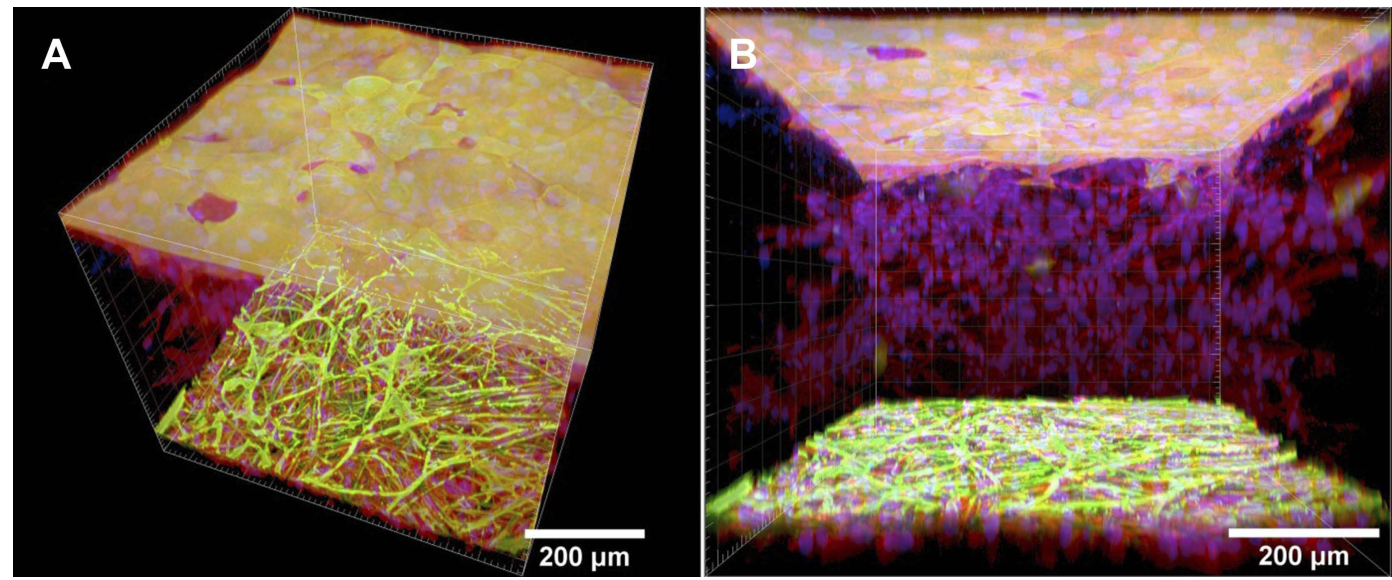

Figure 10 The two-layer construct of skin cells composed of the fibrin-coated PLLA membrane seeded with human dermal fibroblasts, the collagen hydrogel with fibroblasts migrating inside the gel, and the keratinocyte layer on top of the hydrogel. Top-side view of the construct (A), and side-view of the construct (B). The fibroblasts migrated from the membrane into the collagen hydrogel for a period of I 8 days. Keratinocytes proliferated on the hydrogel for I4 days. The PLLA membrane with fibroblasts and the collagen hydrogel with immigrated fibroblasts and keratinocytes on the top of hydrogel were stained separately. Both cell types were stained with phalloidin - TRITC for the cell F-actin cytoskeleton (red), and with Hoechst \#33258 for the cell nuclei (blue). The cytokeratin 14 in the keratinocytes and the fibrin mesh on the nanofibrous membrane (at the bottom) were stained by immunofluorescence (Alexa 488, green). Dragonfly 503 spinning disk confocal microscope with a Zyla 4.2 PLUS sCMOS camera, objective HC PL APO 20x/0.75 IMM CORR CS2.

the other hand, keratinocytes with their apical-basal polarity prefer a $2 \mathrm{D}$ structured surface of the hydrogels. Fujisaki et $\mathrm{al}^{36}$ and many other researchers have observed that collagen hydrogels, mainly collagen IV and collagen I, support the adhesion, proliferation and stratification of keratinocytes. ${ }^{17,36,37}$ In addition, the biosynthesis of ECM molecules by fibroblasts and keratinocytes is enhanced by the physiological conditions of the hydrogels, which is important for hydrogel remodeling and for separating the two cell types by forming the basement membrane. ${ }^{28,38,39}$ Although the encapsulated fibroblasts are separated from the keratinocytes growing on the surface, their communication continues to be mediated by the cytokines and growth factors released from the cells in a paracrine manner, or by cell-hydrogel mechanosensation. ${ }^{40-43}$ The paracrine-based relationship of the cells can be regulated by changing the permeability and the structural properties of the cell-encapsulating substances. For example, Chiu et $\mathrm{al}^{44}$ modulated the permeability of fibrin constructs by varying the concentration of fibrinogen and thrombin. ${ }^{44}$ Bader et $\mathrm{al}^{45}$ changed the keratinocyte-fibroblast paracrine communication by gelatin-based semi-interpenetrating networks. ${ }^{45}$ Moreno-Arotzena et $\mathrm{al}^{46}$ characterized the collagen hydrogel as a biomaterial with a higher void ratio and higher permeability than the fibrin hydrogel. ${ }^{46}$ One of the current approaches to the construction of full-thickness skin substitutes therefore involves embedding dermal fibroblasts into $3 \mathrm{D}$ hydrogels and cultivating epidermal keratinocytes on the surface of hydrogels. Although these 3D co-culture systems have been used for many years for in vitro studies of epithelial-mesenchymal interactions, ${ }^{47}$ for keratinocyte differentiation, ${ }^{36}$ for the dynamics of the basement membrane ${ }^{38}$ and for many other applications, there are many limitations on clinical applications that still need to be overcome.

Although hydrogels have excellent biocompatibility and biodegradability properties for wound healing applications, they are not mechanically stable and their structure tends to be contracted under the traction forces of the embedded cells. ${ }^{27,48}$ The Young's modulus of collagen hydrogels oscillates around units of $\mathrm{kPa}$, depending on temperature, $\mathrm{pH}$, duration of neutralization and many other conditions during polymerization. ${ }^{49,50}$ In order to keep the cells alive during polymerization of the collagen hydrogels, the conditions have to be physiological for the cells, eg $37^{\circ} \mathrm{C}, \mathrm{pH} \sim 7.4$. However, it has previously been observed that the collagen hydrogels formed under physiological conditions are less stiff than those polymerized at basic $\mathrm{pH}^{49,51}$ However, the stiffness of hydrogel fibers plays an important role in cell behavior. Greater stiffness of the fibers suppressed cell adhesion, spreading, proliferation and migration into the hydrogel, due to a lower ability of the cells to transfer their traction forces to the hydrogel fibers. ${ }^{25,52}$

Many approaches leading to improved hydrogel stability and improved mechanical properties have therefore recently been studied. Braziulis et $\mathrm{al}^{21}$ used plastic 
compression for a collagen type I hydrogel. Similarly, Kim et $\mathrm{al}^{53}$ induced structural and mechanical changes to the fibrin-collagen matrix by compressing it. Lotz et $\mathrm{al}^{48}$ reduced the fibroblast-mediated contraction of the collagen hydrogel by cross-linking it with succinimidyl glutarate polyethylene glycol. Another way to improve the mechanical properties of hydrogels is to reinforce them by means of biodegradable synthetic scaffolds. ${ }^{22}$ For example, Franco et $\mathrm{al}^{23}$ combined PCL/PLGA membranes with chitosan-gelatin hydrogels in an optimal ratio to achieve appropriate biocompatibility and mechanical properties for creating a two-layer construct of fibroblasts and keratinocytes. Hartmann-Fritsch et $\mathrm{al}^{31}$ incorporated nanofibrous PLGA membranes or knitted meshes into a bovine collagen I hydrogel with embedded fibroblasts and keratinocytes seeded on the surface. These authors obtained optimal stability of tissue-engineered full-thickness skin analogues by using a knitted mesh, where the epidermal part was well-stratified and the dermal part was revascularized in in vivo experiments. In the present study, we stabilized the collagen hydrogel by underlaying it with a nanofibrous fibrin-coated synthetic biodegradable PLLA membrane, pre-seeded with skin fibroblasts. Our results are in accordance with the recent studies which have shown that the Young's modulus of collagen hydrogels formed in physiological conditions varied around units of $\mathrm{kPa}^{49,51,52}$ The collagen hydrogel disks returned to their original state when compressed at low strain (up to $21 \%$ ). As the scaffolds had a composite character, the mechanical response depended not only on the mechanical properties of the collagen hydrogel but also on the nanofibrous PLLA membrane. The results indicated that collagen hydrogel and PLLA membrane form a serial mechanical connection at compression in which the PLLA membrane start dominating at higher strains (over 56\%). In addition, at higher strains, the collagen hydrogel undergoes structural changes. Pre-seeding the membrane with fibroblasts allowed the fibroblasts later to migrate into the collagen hydrogel without significant contraction of the hydrogel. The stability of the collagen hydrogel can be explained by the optimal ratio between the degradation processes and the remodeling processes mediated by the fibroblasts. During collagen polymerization, the fibroblasts adhering on the membrane were already in their proliferating phase, and they might not generate the strong traction forces that they generate while they are spreading. Moreover, after collagen polymerization, the cells could start migrating into the hydrogel by degrading it with matrix metalloproteases, and by synthesizing their own ECM proteins. ${ }^{16,17}$ In other words, we suppose that the traction forces generated by fibroblasts migrating from the PLLA membrane might be weaker than those generated by fibroblasts directly embedded into the collagen hydrogel. Direct embedding of the fibroblasts into the hydrogel during polymerization can be followed by cell spreading and by generating the intensive cell traction forces that are transmitted into the collagen fibrils. However, fibroblasts migrating from the membrane gradually degrade and remodel the collagen, which enables them to migrate spontaneously into the hydrogel, and leaves the collagen hydrogel relatively unchanged.

In order to increase the attractiveness of the PLLA membrane for the adhesion, spreading and proliferation of the fibroblasts pre-seeded on the membrane, we coated the membrane with a homogeneous nanofibrous fibrin mesh. In our previous study, ${ }^{10}$ we showed that a nanostructured fibrin mesh apparently enhanced fibroblast adhesion, proliferation and biosynthesis of ECM proteins, mainly collagen I and fibronectin. In this study, we have additionally found that the fibrin mesh significantly increased the migration of fibroblasts into the collagen hydrogel in comparison with the non-modified PLLA membrane. Similar results were obtained by $\mathrm{Fu}$ et $\mathrm{al}^{13}$ in experiments with fibrinogen-coated PCL nanofibers. They reported that coating PCL membranes with fibrinogen accelerated the migration of fibroblasts and stimulated their differentiation to myofibroblasts in a transforming growth factor (TGF)- $\beta 1$ rich microenvironment. ${ }^{13}$ In addition, due to the greater adhesion and proliferation of the cells caused by the fibrin mesh, a higher number of cells might migrate into collagen hydrogel. In contrast to our previous work, ${ }^{8}$ we observed that the fibrin mesh under the collagen gel was not degraded, even on day 14 after fibroblast seeding. This may have been caused by focusing the fibroblasts on migrating and on remodeling the 3D collagen hydrogel, rather than focusing on the degradation and remodeling of the fibrin mesh, which was described in detail in our previous studies. ${ }^{8-10}$

In order to create a two-layer cellular skin construct, the collagen hydrogel enriched by spontaneously immigrated fibroblasts was seeded with primary dermal keratinocytes. A monolayer of adhered keratinocytes, with well-developed F-actin and basal cytokeratin 14 filaments, was formed on the whole surface of the collagen hydrogel after 7 days. The cells were able to divide for 14 days of cultivation, and they formed the basal layer with 
highly mitotically active cells and a suprabasal layer with large cells frequently without cell nuclei. Our results correlated with other similar studies focused on the cultivation of keratinocytes on a collagen gel, ${ }^{21,28,31,54}$ or on other forms of collagen substrates. $9,36,55$ The previous studies also reported that a PCL/collagen nanofibrous membrane alone did not stimulate keratinocyte migration, while subsequently coating a membrane composed of an ultrafine fibrous collagen network significantly increased the cell motility. ${ }^{14}$ Our results and other studies have proved that native collagen in the form of a hydrogel provides a physiological 3D microenvironment for the optimal co-cultivation of fibroblasts and keratinocytes. Furthermore, the high void ratio and the high permeability of collagen hydrogels enable paracrine communication between cells. ${ }^{46}$

\section{Conclusion}

We have prepared a two-layer skin construct of fibroblasts and keratinocytes composed of a nanofibrous fibrin-coated PLLA membrane pre-seeded with human dermal fibroblasts, a collagen hydrogel and keratinocytes. The results have shown that the fibroblasts were able to migrate from the membrane upwards into the collagen hydrogel. The PLLA membrane underlying the collagen hydrogel reinforced the whole skin construct, and served as a substrate for the initial attachment, growth and subsequent spontaneous migration of fibroblasts. Moreover, coating the membrane with a nanofibrous fibrin mesh further considerably enhanced the migration of the fibroblasts into the hydrogel. The fibroblasts did not mediate a significant contraction of the collagen hydrogel during their migration, such as has been repeatedly observed when fibroblasts are directly embedded into a collagen hydrogel during the gelling process. The keratinocytes seeded on top of the collagen formed a homogeneous basal layer of proliferating cells. This two-layer skin construct based on a collagen hydrogel with spontaneously immigrated fibroblasts and reinforced by a fibrin-coated nanofibrous membrane seems to be promising for the treatment of extensive full-thickness skin wounds.

\section{Acknowledgments}

We further acknowledge the BioImaging Facility, Institute of Physiology of the Czech Academy of Sciences, Prague, Czech Republic (supported by Czech-BioImaging large RI project No. LM2015062, funded by the Ministry of Education, Youth and Sports, Czech Republic), and the
Light Microscopy Core Facility, Institute of Molecular Genetics of the Czech Academy of Sciences, Prague, Czech Republic (supported by MEYS LM2015062, CZ.02.1.01/0.0/0.0/16_013/0001775, OPPK CZ.2.16/ 3.1.00/21547 and MEYS LO1419), for their support with the $[$ confocal/widefield/superresolution imaging/image analysis] presented here. The group of Dr Tomas Suchy, from Institute of Rock Structure and Mechanics of the Czech Academy of Sciences, is acknowledged for providing us with isolated collagen of type I. Mr Robin Healey (Czech Technical University in Prague) is gratefully acknowledged for his language revision of the manuscript.

\section{Disclosure}

$\mathrm{MB}, \mathrm{JP}, \mathrm{AB}, \mathrm{VJ}$ and $\mathrm{LB}$ received grants from the Grant Agency of the Czech Republic (grant No. 17-0244S). JP received a grant from the Grant Agency of Charles University in Prague (grant No. 756218). AZ and LV received funding from the project "Fighting Infectious Diseases", awarded by the MEYS CR, financed from EFRR (grant No. CZ.02.1.01/0.0/0.0/16_019/0000787). $\mathrm{MB}$ received a postdoctoral fellowship awarded by the Czech Academy of Sciences (reg. No. L200111752). The authors report no other conflicts of interest in this work.

\section{References}

1. Gunter CI, Bader A, Machens H. Regenerative Therapies. In: Steinhoff G, editor. Regenerative Medicine - from Protocol to Patient. 3 ed. Netherlands: Springer International Publishing; 2016:367-386.

2. Duval K, Grover H, Han LH, et al. Modeling physiological events in 2D vs. 3D cell culture. Physiology (Bethesda). 2017;32(4):266-277. doi:10.1152/physiol.00036.2016

3. Ihalainen TO, Aires L, Herzog FA, Schwartlander R, Moeller J, Vogel V. Differential basal-to-apical accessibility of lamin A/C epitopes in the nuclear lamina regulated by changes in cytoskeletal tension. Nat Mater. 2015;14(12):1252-1261. doi:10.1038/nmat4389

4. Ahearne M. Introduction to cell-hydrogel mechanosensing. Interface Focus. 2014;4(2):20130038. doi:10.1098/rsfs.2013.0038

5. Yari A, Teimourian S, Amidi F, et al. The role of biodegradable engineered random polycaprolactone nanofiber scaffolds seeded with nestin-positive hair follicle stem cells for tissue engineering. $A d v$ Biomed Res. 2016;5:22. doi:10.4103/2277-9175.175911

6. Hejazian LB, Esmaeilzade B, Moghanni Ghoroghi F, et al. The role of biodegradable engineered nanofiber scaffolds seeded with hair follicle stem cells for tissue engineering. Iran Biomed J. 2012;16(4):193-201.

7. Chen S, Liu B, Carlson MA, Gombart AF, Reilly DA, Xie J. Recent advances in electrospun nanofibers for wound healing. Nanomedicine (Lond). 2017;12(11):1335-1352. doi:10.2217/nnm-2017-0017

8. Bacakova M, Musilkova J, Riedel T, et al. The potential applications of fibrin-coated electrospun polylactide nanofibers in skin tissue engineering. Int J Nanomedicine. 2016;11:771-789. doi:10.2147/IJN.S99317

9. Bacakova M, Pajorova J, Stranska D, et al. Protein nanocoatings on synthetic polymeric nanofibrous membranes designed as carriers for skin cells. Int J Nanomedicine. 2017;12:1143-1160. doi:10.2147/IJN. S121299 
10. Pajorova J, Bacakova M, Musilkova J, et al. Morphology of a fibrin nanocoating influences dermal fibroblast behavior. Int $J$ Nanomedicine. 2018;13:3367-3380. doi:10.2147/IJN.S162644

11. Zhou T, Wang N, Xue Y, et al. Electrospun tilapia collagen nanofibers accelerating wound healing via inducing keratinocytes proliferation and differentiation. Colloids Surf B Biointerfaces. 2016;143:415-422. doi:10.1016/j.colsurfb.2016.03.052

12. Lai HJ, Kuan $\mathrm{CH}$, Wu HC, et al. Tailored design of electrospun composite nanofibers with staged release of multiple angiogenic growth factors for chronic wound healing. Acta Biomater. 2014;10 (10):4156-4166. doi:10.1016/j.actbio.2014.05.001

13. Fu X, Xu M, Jia C, et al. Differential regulation of skin fibroblasts for their TGF- $\beta 1$-dependent wound healing activities by biomimetic nanofibers. $J$ Mater Chem B. 2016;4(31):5246-5255. doi:10.1039/C6TB00882H

14. Fu X, Xu M, Liu J, Qi Y, Li S, Wang H. Regulation of migratory activity of human keratinocytes by topography of multiscale collagen-containing nanofibrous matrices. Biomaterials. 2014;35 (5):1496-1506. doi:10.1016/j.biomaterials.2013.11.013

15. Mahjour SB, Fu X, Yang X, Fong J, Sefat F, Wang H. Rapid creation of skin substitutes from human skin cells and biomimetic nanofibers for acute full-thickness wound repair. Burns. 2015;41(8):1764-1774. doi:10.1016/j.burns.2015.06.011

16. Miron-Mendoza M, Lin X, Ma L, Ririe P, Petroll WM. Individual versus collective fibroblast spreading and migration: regulation by matrix composition in 3D culture. Exp Eye Res. 2012;99:36-44.

17. Sriram G, Bigliardi PL, Bigliardi-Qi M. in vitro. Eur J Cell Biol. 2015;94(11):483-512. doi:10.1016/j.ejcb.2015.08.001

18. Chaudhari AA, Vig K, Baganizi DR, et al. Future prospects for scaffolding methods and biomaterials in skin tissue engineering: a review. Int J Mol Sci. 2016;17(12):1974. doi:10.3390/ijms17121974

19. Shang J, Theato P. Smart composite hydrogel with $\mathrm{pH}$-, ionic strength- and temperature-induced actuation. Soft Matter. 2018. doi:10.1039/C8SM01728J

20. Jhon MS, Andrade JD. Water and hydrogels. J Biomed Mater Res. 1973;7(6):509-522. doi:10.1002/jbm.820070604

21. Braziulis E, Diezi M, Biedermann T, et al. Modified plastic compression of collagen hydrogels provides an ideal matrix for clinically applicable skin substitutes. Tissue Eng Part C Methods. 2012;18 (6):464-474. doi:10.1089/ten.TEC.2011.0561

22. Lee S, Kim HS, Yoo HS. Electrospun nanofibrils embedded hydrogel composites for cell cultivation in a biomimetic environment. RSC Adv. 2017;7(85):54246-54253. doi:10.1039/C7RA08595H

23. Franco RA, Nguyen TH, Lee BT. Preparation and characterization of electrospun PCL/PLGA membranes and chitosan/gelatin hydrogels for skin bioengineering applications. J Mater Sci Mater Med. 2011;22 (10):2207-2218. doi:10.1007/s10856-011-4402-8

24. Huang J, Ren J, Chen G, et al. Tunable sequential drug delivery system based on chitosan/hyaluronic acid hydrogels and PLGA microspheres for management of non-healing infected wounds. Mater Sci Eng C Mater Biol Appl. 2018;89:213-222. doi:10.1016/j. msec.2018.04.009

25. Nam K, Sakai Y, Hashimoto Y, Kimura T, Kishida A. Fabrication of a heterostructural fibrillated collagen matrix for the regeneration of soft tissue function. Soft Matter. 2012;8(2):472-480. doi:10.1039/ C1SM06543B

26. Branco Da Cunha C, Klumpers DD, Li WA, et al. Influence of the stiffness of three-dimensional alginate/collagen-I interpenetrating networks on fibroblast biology. Biomaterials. 2014;35(32):89278936. doi:10.1016/j.biomaterials.2014.06.047

27. Polio SR, Smith ML. Patterned hydrogels for simplified measurement of cell traction forces. Methods Cell Biol. 2014;121:17-31. doi:10.1016/B978-0-12-800281-0.00002-6

28. El Ghalbzouri A, Commandeur S, Rietveld MH, Mulder AA, Willemze R. Replacement of animal-derived collagen matrix by human fibroblastderived dermal matrix for human skin equivalent products. Biomaterials. 2009;30(1):71-78. doi:10.1016/j.biomaterials.2008.09.002
29. Jiang H, Grinnell F. Cell-matrix entanglement and mechanical anchorage of fibroblasts in three-dimensional collagen matrices. Mol Biol Cell. 2005;16(11):5070-5076.

30. Grinnell F. Fibroblast biology in three-dimensional collagen matrices. Trends Cell Biol. 2003;13(5):264-269.

31. Hartmann-Fritsch F, Biedermann T, Braziulis E, et al. Collagen hydrogels strengthened by biodegradable meshes are a basis for dermo-epidermal skin grafts intended to reconstitute human skin in a one-step surgical intervention. J Tissue Eng Regen Med. 2016;10 (1):81-91. doi:10.1002/term.1665

32. Delvoye P, Wiliquet P, Leveque JL, Nusgens BV, Lapiere CM. Measurement of mechanical forces generated by skin fibroblasts embedded in a three-dimensional collagen gel. $J$ Invest Dermatol. 1991;97(5):898-902.

33. Legant WR, Miller JS, Blakely BL, Cohen DM, Genin GM, Chen CS. Measurement of mechanical tractions exerted by cells in threedimensional matrices. Nat Methods. 2010;7(12):969-971. doi:10.1038/nmeth.1531

34. Riedel T, Brynda E, Dyr JE, Houska M. Controlled preparation of thin fibrin films immobilized at solid surfaces. J Biomed Mater Res A. 2009;88(2):437-447. doi:10.1002/jbm.a.31755

35. Arnette C, Koetsier JL, Hoover P, Getsios S, Green KJ. In vitro model of the epidermis: connecting protein function to 3D structure. Methods Enzymol. 2016;569:287-308. doi:10.1016/bs.mie.2015. 07.015

36. Fujisaki H, Adachi E, Hattori S. Keratinocyte differentiation and proliferation are regulated by adhesion to the three-dimensional meshwork structure of type IV collagen. Connect Tissue Res. 2008;49(6):426-436. doi:10.1080/03008200802324998

37. Klar AS, Michalak K, Bottcher-Haberzeth S, Reichmann E, Meuli M, Biedermann T. The expression pattern of keratin 24 in tissue-engineered dermo-epidermal human skin substitutes in an in vivo model. Pediatr Surg Int. 2018;34(2):237-244. doi:10.1007/s00383-017-4198-9

38. Marionnet C, Pierrard C, Vioux-Chagnoleau C, Sok J, Asselineau D, Bernerd $\mathrm{F}$. Interactions between fibroblasts and keratinocytes in morphogenesis of dermal epidermal junction in a model of reconstructed skin. $J$ Invest Dermatol. 2006;126(5):971-979. doi:10.1038/sj.jid.5700230

39. Breitkreutz D, Koxholt I, Thiemann K, Nischt R. Skin basement membrane: the foundation of epidermal integrity-BM functions and diverse roles of bridging molecules nidogen and perlecan. Biomed Res Int. 2013;2013:179784. doi:10.1155/2013/179784

40. Tuan TL, Keller LC, Sun D, Nimni ME, Cheung D. Dermal fibroblasts activate keratinocyte outgrowth on collagen gels. J Cell Sci. 1994;107(Pt 8):2285-2289.

41. Wojtowicz AM, Oliveira S, Carlson MW, Zawadzka A, Rousseau CF, Baksh D. The importance of both fibroblasts and keratinocytes in a bilayered living cellular construct used in wound healing. Wound Repair Regen. 2014;22(2):246-255. doi:10.1111/wrr.12154

42. Doyle AD, Yamada KM. Mechanosensing via cell-matrix adhesions in 3D microenvironments. Exp Cell Res. 2016;343(1):60-66. doi:10.1016/j.yexcr.2015.10.033

43. Stunova A, Vistejnova L. Dermal fibroblasts-A heterogeneous population with regulatory function in wound healing. Cytokine Growth Factor Rev. 2018;39:137-150. doi:10.1016/j.cytogfr.2018.01.003

44. Chiu CL, Hecht V, Duong H, Wu B, Tawil B. Permeability of threedimensional fibrin constructs corresponds to fibrinogen and thrombin concentrations. Biores Open Access. 2012;1(1):34-40. doi:10.1089/ biores.2012.0211

45. Bader RA, Kao WJ. Modulation of the keratinocyte-fibroblast paracrine relationship with gelatin-based semi-interpenetrating networks containing bioactive factors for wound repair. J Biomater Sci Polym Ed. 2009;20(7-8):1005-1030. doi:10.1163/156856209X444402

46. Moreno-Arotzena O, Meier JG, Del Amo C, Garcia-Aznar JM. Characterization of fibrin and collagen gels for engineering wound healing models. Materials (Basel). 2015;8(4):1636-1651. doi:10.3390/ma8041636 
47. Maas-Szabowski N, Shimotoyodome A, Fusenig NE. Keratinocyte growth regulation in fibroblast cocultures via a double paracrine mechanism. J Cell Sci. 1999;112(Pt 12):1843-1853.

48. Lotz C, Schmid FF, Oechsle E, Monaghan MG, Walles H, GroeberBecker F. Cross-linked collagen hydrogel matrix resisting contraction to facilitate full-thickness skin equivalents. ACS Appl Mater Interfaces. 2017;9(24):20417-20425. doi:10.1021/acsami.7b04017

49. Achilli M, Mantovani D. Tailoring mechanical properties of collagenbased scaffolds for vascular tissue engineering: the effects of $\mathrm{pH}$, temperature and ionic strength on gelation. Polymers. 2010;2:4. doi:10.3390/polym2040664

50. Lopez-Garcia MD, Beebe DJ, Crone WC. Mechanical interactions of mouse mammary gland cells with collagen in a three-dimensional construct. Ann Biomed Eng. 2010;38(8):2485-2498. doi:10.1007/s10439010-0015-5

51. Antoine EE, Vlachos PP, Rylander MN. Tunable collagen I hydrogels for engineered physiological tissue micro-environments. PLoS One. 2015;10:3. doi:10.1371/journal.pone. 0122500
52. Xie J, Bao M, Bruekers SMC, Huck WTS. Collagen gels with different fibrillar microarchitectures elicit different cellular responses. ACS Appl Mater Interfaces. 2017;9(23):19630-19637. doi:10.1021/ acsami.7b03883

53. Kim OV, Litvinov RI, Chen J, Chen DZ, Weisel JW, Alber MS. Compression-induced structural and mechanical changes of fibrin-collagen composites. Matrix Biol. 2017;60-61:141-156. doi:10.1016/j. matbio.2016.10.007

54. Stark H-J, Szabowski A, Fusenig NE, Maas-Szabowski N. Organotypic cocultures as skin equivalents: a complex and sophisticated in vitro system. Biol Proced Online. 2004;6:55-60. doi:10.1251/bpo72

55. Benny P, Badowski C, Lane EB, Raghunath M. Making more matrix: enhancing the deposition of dermal-epidermal junction components in vitro and accelerating organotypic skin culture development, using macromolecular crowding. Tissue Eng Part A. 2015;21(1-2):183192. doi:10.1089/ten.TEA.2013.0784 


\section{Supplementary materials}

Video SI The horizontal sections through the collagen hydrogel with keratinocytes adhered on the hydrogel surface and fibroblasts homogeneously immigrated inside the collagen hydrogel. The fibroblasts migrated from the membrane into the collagen hydrogel for a period of 11 days. Keratinocytes proliferated on the hydrogel for 7 days. Both cell types were stained with phalloidin-TRITC for the cell F-actin cytoskeleton (red), and with Hoechst \#33258 for the cell nuclei (blue). The cytokeratin 14 in the keratinocytes was stained by immunofluorescence (Alexa 488, green). Dragonfly 503 spinning disk confocal microscope with a Zyla 4.2 PLUS sCMOS camera, objective HC PL APO 20x/0.75 IMM CORR CS2.

Video S2 This video shows the two-layer construct of skin cells, depicted in Figure 10, in dynamic mode. The construct was composed of the fibrin-coated PLLA membrane seeded with human dermal fibroblasts, the collagen hydrogel with fibroblasts migrating inside the gel, and the keratinocyte layer on top of hydrogel. The fibroblasts migrated from the membrane into the collagen hydrogel for a period of 18 days. Keratinocytes proliferated on the hydrogel for 14 days. The PLLA membrane with fibroblasts and the collagen hydrogel with immigrated fibroblasts and keratinocytes on the top of hydrogel were stained separately. Both cell types were stained with phalloidin - TRITC for the cell F-actin cytoskeleton (red), and with Hoechst \#33258 for the cell nuclei (blue). The cytokeratin 14 in the keratinocytes and the fibrin mesh on the nanofibrous membrane (at the bottom) were stained by immunofluorescence (Alexa 488, green). Dragonfly 503 spinning disk confocal microscope with a Zyla 4.2 PLUS sCMOS camera, objective HC PL APO 20x/0.75 IMM CORR CS2.

\section{Publish your work in this journal}

The International Journal of Nanomedicine is an international, peerreviewed journal focusing on the application of nanotechnology in diagnostics, therapeutics, and drug delivery systems throughout the biomedical field. This journal is indexed on PubMed Central, MedLine, CAS, SciSearch ${ }^{\circledR}$, Current Contents ${ }^{\circledR} /$ Clinical Medicine, $^{\circ}$
Journal Citation Reports/Science Edition, EMBase, Scopus and the Elsevier Bibliographic databases. The manuscript management system is completely online and includes a very quick and fair peer-review system, which is all easy to use. Visit http://www.dovepress.com/ testimonials.php to read real quotes from published authors. 